\title{
Three-component coupling of benzyne: Domino intermolecular carbopalladation
}

\author{
Jaclyn L. Henderson, Andrew S. Edwards and Michael F. Greaney* \\ University of Edinburgh, School of Chemistry, Joseph Black Building, King's Buildings, West Mains \\ Road, Edinburgh, EH9 3JJ, UK \\ Organon Laboratories Ltd., Newhouse, Lanarkshire, ML1 5SH, UK \\ Michael.Greaney@ed.ac.uk
}

Supporting Information

\section{Part A: Experimental Procedures}

\section{General}

NMR spectra were recorded on a Brüker dpx360 (360 MHz) instrument and calibrated to residual solvent peaks: proton $\left(\mathrm{CDCl}_{3} 7.26 \mathrm{ppm}\right.$ or DMSO $\left.2.50 \mathrm{ppm}\right)$ and carbon $\left(\mathrm{CDCl}_{3} 77.0 \mathrm{ppm}\right.$ or DMSO $39.4 \mathrm{ppm})$. The ${ }^{1} \mathrm{H}$ data is presented as follows: chemical shift (in ppm on the $\delta$ scale), multiplicity ( $\mathrm{s}=$ singlet, $\mathrm{d}=$ =doublet, $\mathrm{t}=$ triplet, $\mathrm{q}=$ quartet, $\mathrm{m}=$ multiplet), the coupling constant $(\mathrm{J}$, in Hertz) and integration. The ${ }^{13} \mathrm{C}$ data is reported as the ppm on the $\delta$ scale followed by the interpretation. IR spectra were recorded on a JASCO FT/IR-460 plus instrument using $4 \mathrm{~mm}$ sodium chloride disks. The wavelengths of the maximum absorbance $\left(v_{\max }\right)$ are quoted in $\mathrm{cm}^{-1}$. Electrospray high resolution mass spectrometry was performed by the EPSRC National Mass Spectrometry Service Centre, Swansea, using a Finnigan MAT 900 XLT double focusing mass spectrometer and at Organon Laboratories using an Applied Biosystems Mariner time of flight mass spectrometer. The data is recorded as the ionisation method followed by the calculated and measured masses. TLC was performed on Merck 60F254 silica plates and visualised by UV light and/or anisaldehyde or potassium permanganate stains. The compounds were purified by wet flash chromatography using Merck Kieselgel 60 (particle size 35-70) silica under a positive pressure. The eluent is quoted as a percentage. All solvents were dried before use unless otherwise stated. DME was distilled over sodium with benzophenone as an indicator. DCM was distilled over calcium hydride. 4-methyl-2-(trimethylsilyl)phenyl trifluoromethanesulfonate was prepared according to published procedure. ${ }^{1}$ All other chemicals were purchased from a chemical supplier and used as received.

\footnotetext{
${ }^{1}$ Peña, D.; Cobas, A.; Pérez, D.; Guitián, E. Synthesis 2002, 1454-1458.
} 


\section{TCC of allyl chloride, benzyne and methyl acrylate}

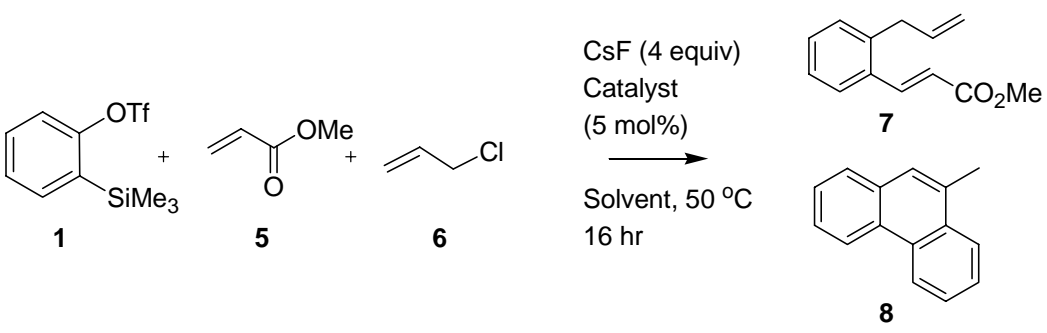

\section{Catalyst Screen}

\begin{tabular}{|c|c|c|c|}
\hline Entry $^{a}$ & Pd source & Ligand & Yield of 7 or $8(\%)$ \\
\hline 1 & $\mathrm{Pd}_{2}(\mathrm{dba})_{3}$ & dppe & 7,9 \\
\hline 2 & $\mathrm{Pd}\left(\mathrm{PPh}_{3}\right)_{4}$ & & 7,18 \\
\hline 3 & $\mathrm{Pd}(\mathrm{OAc})_{2}$ & $\mathrm{PPh}_{3}$ & 7,17 \\
\hline 4 & $\mathrm{Pd}(\mathrm{OAc})_{2}$ & dppe & 7,$28 ; \mathbf{8}, 58$ \\
\hline 5 & $\mathrm{Pd}(\mathrm{OAc})_{2}$ & $\mathrm{P}(\mathrm{Cy})_{3}$ & 0 \\
\hline 6 & $\mathrm{Pd}(\mathrm{OAc})_{2}$ & dppp & Mainly phenanthrene \\
\hline 7 & $\mathrm{PdCl}_{2}(\mathrm{CN})_{2}$ & & 0 \\
\hline 8 & $\mathrm{Pd}(\mathrm{dppf}) \mathrm{Cl}_{2}$ & & 0 (complex mixture) \\
\hline 9 & $\mathrm{Pd}\left(\mathrm{PPh}_{3}\right)_{2} \mathrm{Cl}_{2}$ & & Mainly phenanthrene \\
\hline
\end{tabular}

${ }^{a}$ All reactions carried out in acetonitrile.

\section{Solvent Screen}

\begin{tabular}{cccc}
\hline Entry $^{a}$ & Solvent & Ratio 7:8 & Yield (\%) \\
\hline 1 & MeCN & & 28 \\
2 & MeCN/DCM & $1: 1$ & 24 \\
3 & MeCN/Toluene & $1: 1$ & 14 \\
4 & MeCN/1,4-dioxane & $1: 2$ & 34 \\
5 & DME & & 50 \\
6 & 1,4-dioxane & & Starting material \\
7 & DME/1,4-dioxane & $1: 1$ & Starting material \\
8 & THF & & $0^{c}$ \\
9 & DMF & & $0^{c}$ \\
\hline
\end{tabular}

${ }^{a}$ Catalyst system: $\mathrm{Pd}(\mathrm{OAc})_{2} / \mathrm{dppe} 5 \mathrm{~mol} \%{ }^{b}{ }^{b} \mathrm{MeCN}$ added slowly over 6 hours. ${ }^{c}$ Neither product or starting material evident in crude NMR

\section{TCC of benzyne, allyl chloride and methyl acrylate}

Methyl acrylate ( $0.49 \mathrm{mmol}, 2.4$ equiv) and allyl chloride $(0.25 \mathrm{mmol}, 1.2$ equiv) were added to a stirred suspension of $\mathrm{Pd}(\mathrm{OAc})_{2}(4 \mathrm{mg}, 5 \mathrm{~mol} \%$ ), dppe (4 mg, $5 \mathrm{~mol} \%$ ) and $\mathrm{CsF}$ (4 equiv) in freshly distilled DME (1.5 $\mathrm{mL}$ ) under $\mathrm{N}_{2}$ in a $5 \mathrm{~mL}$ round bottomed flask. This was followed by the dropwise addition of 2(trimethylsilyl)phenyl trifluoromethane sulfonate (1) $\left(0.21 \mathrm{mmol}, 1\right.$ equiv). The reaction was heated to $50{ }^{\circ} \mathrm{C}$ and stirred overnight. The reaction mixture was diluted with DCM and filtered through a short plug of silica then concentrated under reduced pressure. Purification by flash column chromatography on silica gel, eluting with $10 \%$ EtOAc/hexanes yielded the title compound.

(E)-methyl 3-(2-allylphenyl)acrylate (7): Isolated as a colourless oil in a yield of $50 \% .{ }^{1} \mathrm{H}$ NMR $\left(\mathrm{CDCl}_{3}, 360\right.$ MHz) $\delta 8.06(\mathrm{~d}, 1 \mathrm{H}, \mathrm{J}=15.8), 7.58(\mathrm{dd}, 1 \mathrm{H}, \mathrm{J}=7.5,1.4), 7.33(\mathrm{dd}, 1 \mathrm{H}, \mathrm{J}=7.2,1.4), 7.32-7.25(\mathrm{~m}, 2 \mathrm{H}), 6.37$ $(\mathrm{d}, 1 \mathrm{H}, \mathrm{J}=15.8), 5.96(\mathrm{~m}, 1 \mathrm{H}), 5.08(\mathrm{dd}, 1 \mathrm{H}, \mathrm{J}=10.1,1.6), 5.00(\mathrm{dd}, 1 \mathrm{H}, \mathrm{J}=15.3,1.7), 3.83(\mathrm{~s}, 3 \mathrm{H}), 3.58(\mathrm{~d}$, 
$2 \mathrm{H}, \mathrm{J}=7.7) ;{ }^{13} \mathrm{C} \mathrm{NMR}\left(\mathrm{CDCl}_{3}, 62.9 \mathrm{MHz}\right) \delta 167.4$ (quat), $142.4(\mathrm{CH}), 139.2$ (quat), $136.5(\mathrm{CH}), 133.3$ (quat), $130.3(\mathrm{CH}), 130.2(\mathrm{CH}), 126.8(\mathrm{CH}), 126.6(\mathrm{CH}), 119.1(\mathrm{CH}), 116.4\left(\mathrm{CH}_{2}\right), 51.7\left(\mathrm{CH}_{3}\right), 37.4\left(\mathrm{CH}_{2}\right) ; \mathrm{IR}$ $\left(\right.$ film $\left./ \mathrm{cm}^{-1}\right) 2949,1717,1634,1434,1318,1272,1194,1171 ; \mathrm{HRMS}\left(\mathrm{ES}^{+}\right)$cald. for $\mathrm{C}_{13} \mathrm{H}_{15} \mathrm{O}_{2}:(\mathrm{M}+\mathrm{H})^{+}$ 203.1067; found 203.1067.

\section{TCC of methyl bromoacetate, benzyne and acrylates}

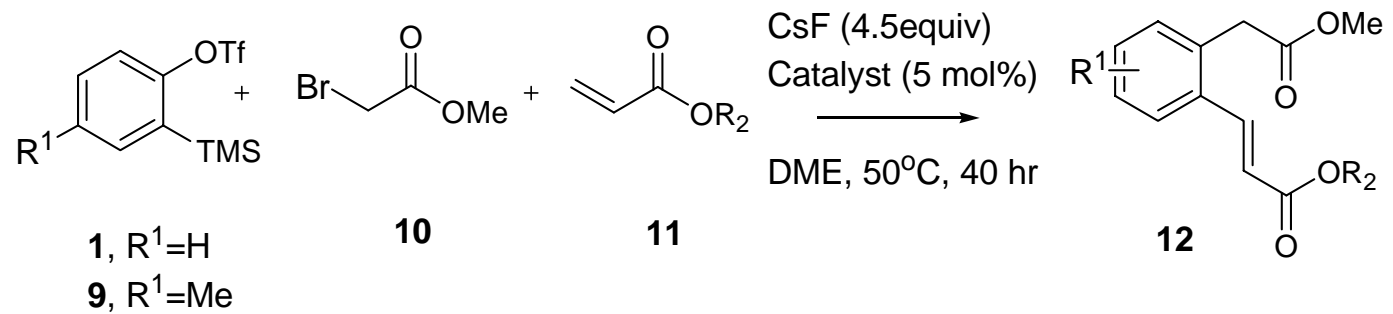

\section{Catalyst Screen}

\begin{tabular}{|c|c|c|c|}
\hline Entry & Pd Source & Ligand & Yield of $12(\%)$ \\
\hline 1 & $\mathrm{Pd}(\mathrm{OAc})_{2}$ & dppe & 30 \\
\hline 2 & $\mathrm{Pd}\left(\mathrm{PPh}_{3}\right)_{4}$ & & 51 \\
\hline 3 & $\mathrm{Pd}_{2}(\mathrm{dba})_{3}$ & dpppent & 60 \\
\hline 4 & $\mathrm{Pd}_{2}(\mathrm{dba})_{3}$ & dppf & Complex mix \\
\hline 5 & $\mathrm{Pd}(\mathrm{dppf}) \mathrm{Cl}_{2}$ & & 75 \\
\hline 6 & $\mathrm{Pd}(\mathrm{dppf}) \mathrm{Cl}_{2}$ & dppf & 39 \\
\hline 7 & $\mathrm{Pd}_{2}(\mathrm{dba})_{3}$ & dppp & 65 \\
\hline 8 & $\mathrm{Pd}_{2}(\mathrm{dba})_{3}$ & dppb & 57 \\
\hline 9 & $\mathrm{Pd}_{2}(\mathrm{dba})_{3}$ & dpphex & 29 \\
\hline 10 & $\mathrm{Pd}_{2}(\mathrm{dba})_{3}$ & dppm & Complex mix \\
\hline 11 & $\mathrm{Pd}(\mathrm{OAc})_{2}$ & dppb & 42 \\
\hline 12 & $\mathrm{Pd}_{2}(\mathrm{dba})_{3}$ & dppe & 44 \\
\hline 13 & $\mathrm{Pd}(\mathrm{dppe})_{2}$ & & 31 \\
\hline
\end{tabular}

TCC of $\mathbf{1}, \mathbf{1 0}$ and methyl acrylate was carried out in $\mathrm{MeCN}$ with $\mathrm{Pd}(\mathrm{dppf}) \mathrm{Cl}_{2}$ as catalyst. None of the desired product, 12a, could be isolated.

\section{General method for TCC of benzyne, bromoacetates and acrylates}

Methyl acrylate $(0.31 \mathrm{mmol}, 1.5$ equiv) and methyl bromoacetate, 10, $(0.21 \mathrm{mmol}, 1$ equiv $)$ were added to a stirred suspension of $\mathrm{Pd}(\mathrm{dppf}) \mathrm{Cl}_{2}(8.4 \mathrm{mg}, 5 \mathrm{~mol} \%)$ and $\mathrm{CsF}$ (4.5 equiv) in freshly distilled DME (1 ml) under $\mathrm{N}_{2}$. This was followed by the dropwise addition of 2-(trimethylsilyl)phenyl trifluoromethane sulfonate (1) (0.31 mmol, 1.5 equiv). The reaction was stirred at $50{ }^{\circ} \mathrm{C}$ over two nights, after which time it was diluted with DCM and filtered through a short plug of silica then concentrated under reduced pressure. Flash chromatography on silica gel, eluting with $20 \%$ EtOAc/hexanes yielded the title compounds.

(E)-methyl 3-(2-((methoxycarbonyl)methyl)phenyl)acrylate (12a): Isolated as a colourless oil in a yield of 75\%. ${ }^{1} \mathrm{H} \mathrm{NMR}\left(\mathrm{CDCl}_{3}, 360 \mathrm{MHz}\right) \delta 7.96(\mathrm{~d}, 1 \mathrm{H}, \mathrm{J}=15.8), 7.60(\mathrm{dd}, 1 \mathrm{H}, \mathrm{J}=1.6,7.3), 7.38-7.27(\mathrm{~m}, 3 \mathrm{H}), 6.38$ $(\mathrm{d}, 1 \mathrm{H}, \mathrm{J}=15.8), 3.81(\mathrm{~s}, 3 \mathrm{H}), 3.81(\mathrm{~s}, 2 \mathrm{H}), 3.70(\mathrm{~s}, 3 \mathrm{H}) ;{ }^{13} \mathrm{C} \mathrm{NMR}\left(\mathrm{CDCl}_{3}, 90.55 \mathrm{MHz}\right) \delta 171.3$ (quat), 167.1 (quat), $141.8(\mathrm{CH}), 133.9$ (quat), 133.5 (quat), $131.1(\mathrm{CH}), 130.1(\mathrm{CH}), 127.9(\mathrm{CH}), 126.9(\mathrm{CH}), 120.0(\mathrm{CH})$, $52.2\left(\mathrm{CH}_{3}\right), 51.7\left(\mathrm{CH}_{3}\right), 38.6\left(\mathrm{CH}_{2}\right)$; IR $\left(\mathrm{film} / \mathrm{cm}^{-1}\right) 2952,1716,1634,1434,1319,1170$; HRMS (ES $\left.{ }^{+}\right)$cald. for $\mathrm{C}_{13} \mathrm{H}_{15} \mathrm{O}_{4}:(\mathrm{M}+\mathrm{H})^{+}$235.0965. Found: 235.0967.

(E)-tert-butyl 3-(2-((methoxycarbonyl)methyl)phenyl)acrylate (12b): Isolated as a colourless oil in a yield of 80\%. ${ }^{1} \mathrm{H}$ NMR $\left(\mathrm{CDCl}_{3}, 360 \mathrm{MHz}\right) \delta 7.86(\mathrm{~d}, 1 \mathrm{H}, \mathrm{J}=15.7), 7.59(\mathrm{~m}, 1 \mathrm{H}), 7.37-7.25(\mathrm{~m}, 3 \mathrm{H}), 6.31(\mathrm{~d}, 2 \mathrm{H}, \mathrm{J}=$ 
15.7), $3.78(\mathrm{~s}, 2 \mathrm{H}), 3.70(\mathrm{~s}, 3 \mathrm{H}), 1.52(\mathrm{~s}, 9 \mathrm{H}) ;{ }^{13} \mathrm{C} \mathrm{NMR}\left(\mathrm{CDCl}_{3}, 90.55 \mathrm{MHz}\right) \delta 171.3$ (quat), 165.9 (quat), 140.3 $(\mathrm{CH}), 134.0$ (quat), 133.2 (quat), $130.8(\mathrm{CH}), 129.7(\mathrm{CH}), 127.6(\mathrm{CH}), 126.7(\mathrm{CH}), 122.2(\mathrm{CH}), 80.4($ quat), 52.0 $\left(\mathrm{CH}_{3}\right), 38.4\left(\mathrm{CH}_{2}\right), 28.0\left(\mathrm{CH}_{3}\right)$; IR (film/ $\left.\mathrm{cm}^{-1}\right)$ 2978, 1740, 1708, 1633, 1321, 1151; HRMS (ES $)^{+}$cald. for $\mathrm{C}_{16} \mathrm{H}_{21} \mathrm{O}_{4}:(\mathrm{M}+\mathrm{H})^{+}$277.1434. Found: 277.1435.

(E)-tert-butyl 3-(2-((methoxycarbonyl)methyl)-4-methylphenyl)acrylate (12c) and (E)-tert-butyl 3-(2((methoxycarbonyl)methyl)-5-methylphenyl)acrylate (12d): Isolated as a colourless oil in a yield of $70 \%$. ${ }^{1} \mathrm{H}$ $\operatorname{NMR}\left(\mathrm{CDCl}_{3}, 360 \mathrm{MHz}\right) \delta 7.75(\mathrm{~d}, 1 \mathrm{H}, \mathrm{J}=15.7), 7.42(\mathrm{~d}, 0.5 \mathrm{H}, \mathrm{J}=7.9), 7.34(\mathrm{~s}, 0.5 \mathrm{H}), 7.07(\mathrm{~m}, 3 \mathrm{H}), 6.22(\mathrm{~d}$, $0.5 \mathrm{H}, \mathrm{J}=15.7), 6.20(\mathrm{~d}, 0.5 \mathrm{H}, \mathrm{J}=15.7), 3.66(\mathrm{~s}, 1 \mathrm{H}), 3.64(\mathrm{~s}, 1 \mathrm{H}), 3.63(\mathrm{~s}, 1.5 \mathrm{H}), 3.61(\mathrm{~s}, 1.5 \mathrm{H}), 2.27(\mathrm{~s}, 3 \mathrm{H})$, $1.44(\mathrm{~s}, 9 \mathrm{H}) ;{ }^{13} \mathrm{C} \mathrm{NMR}\left(\mathrm{CDCl}_{3}, 90.55 \mathrm{MHz}\right) \delta 171.6$ (quat), 171.6 (quat), 166.2 (quat), 166.1 (quat), $140.6(\mathrm{CH})$, $140.3(\mathrm{CH}), 140.2$ (quat), 137.4 (quat), 133.8 (quat), 133.3 (quat), $131.7(\mathrm{CH}), 131.2$ (quat), $130.9(\mathrm{CH}), 130.7$ $(\mathrm{CH}), 130.5$ (quat), $128.6(\mathrm{CH}), 127.4(\mathrm{CH}), 126.7(\mathrm{CH}), 122.0(\mathrm{CH}), 121.2(\mathrm{CH}), 80.5$ (quat), 80.4 (quat), 52.2 $\left(\mathrm{CH}_{3}\right), 52.1\left(\mathrm{CH}_{3}\right), 38.5\left(\mathrm{CH}_{2}\right), 38.1\left(\mathrm{CH}_{2}\right), 28.1\left(\mathrm{CH}_{3}\right), 21.2\left(\mathrm{CH}_{3}\right), 21.0\left(\mathrm{CH}_{3}\right) ; \mathrm{IR}\left(\mathrm{film} / \mathrm{cm}^{-1}\right) 2978,1741,1707$, $1633,1321,1251,1151$; HRMS $\left(\mathrm{ES}^{+}\right)$cald for $\mathrm{C}_{13} \mathrm{H}_{13} \mathrm{O}_{3}:\left(\mathrm{M}-\mathrm{O}^{\mathrm{t}} \mathrm{Bu}\right)^{+}$217.0859. Found: 217.0852 .

\section{TCC of benzyl bromides, benzyne and tert-butyl acrylate}

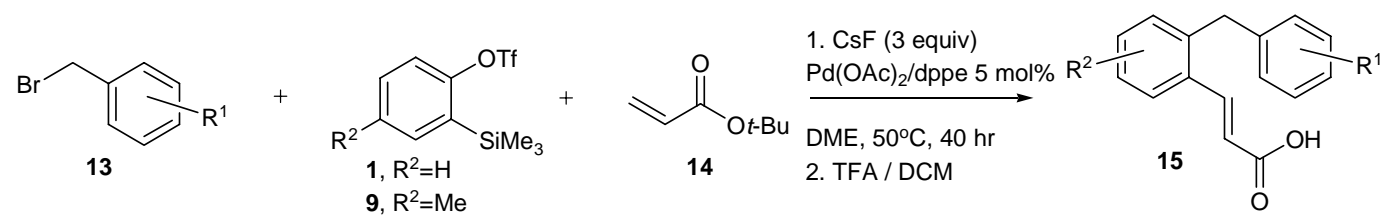

Catalyst Screen

\begin{tabular}{ccccc}
\hline Entry & $\begin{array}{c}\text { Palladium } \\
\text { Source }\end{array}$ & Ligand & $\begin{array}{c}\text { Volume } \\
(\mathrm{mL})\end{array}$ & Outcome \\
\hline 1 & $\mathrm{Pd}(\mathrm{dppf}) \mathrm{Cl}_{2}$ & & 1 & $1: 1 \mathbf{1 5}$ with phenanthrene \\
2 & $\mathrm{Pd}(\mathrm{OAc})_{2}$ & dppe & 1 & $64 \%$ yield of $\mathbf{1 5}$ \\
3 & $\mathrm{Pd}_{2}(\mathrm{dba})_{3}$ & dppe & 1 & $47 \%$ yield of $\mathbf{1 5}$ \\
4 & $\mathrm{Pd}(\mathrm{OAc})_{2}$ & dppe & 15 & Complex mix \\
5 & $\mathrm{Pd}(\mathrm{OAc})_{2}$ & $\mathrm{dppb}$ & 1 & $20 \%$ yield of $\mathbf{1 5}$ \\
6 & $\mathrm{Pd}(\mathrm{OAc})_{2}$ & dppp & 1 & Complex mix \\
7 & $\mathrm{Pd}(\mathrm{OAc})_{2}$ & xantphos & 1 & $0 \%$ yield of 15 \\
8 & $\mathrm{Pd}\left(\mathrm{PPh}_{3}\right)_{4}$ & & 1 & Complex mix \\
\hline
\end{tabular}

TCC of 13b, 1 and 14 was also carried out in $\mathrm{MeCN}$ using the $\mathrm{Pd}(\mathrm{OAc})_{2} /$ dppe catalyst system. Product $\mathbf{1 5 b}$ was isolated in a yield of $27 \%$.

\section{General method for TCC of benzyl bromides, benzyne and acrylates}

Benzyl bromide ( $0.31 \mathrm{mmol}, 1.5$ equiv) and tert-butyl acrylate $(0.21 \mathrm{mmol}, 1$ equiv) were added to a stirred suspension of $\mathrm{Pd}(\mathrm{OAc})_{2}(2.4 \mathrm{mg}, 5 \mathrm{~mol} \%)$, dppe (4.3 mg, $5 \mathrm{~mol} \%$ ) and $\mathrm{CsF}$ (3 equiv) in freshly distilled DME $(1 \mathrm{ml})$ under $\mathrm{N}_{2}$. This was followed by the dropwise addition of 2-(trimethylsilyl)phenyl trifluoromethane sulfonate (1) $\left(0.21 \mathrm{mmol}, 1\right.$ equiv). The reaction was stirred at $50{ }^{\circ} \mathrm{C}$ over 2 nights. The reaction mixture was diluted with DCM and filtered through a pad of silica. Purification was achieved either by a) chromatography on silica gel eluting with EtOAc/hexanes to produce the ester, or b) treatment with TFA $(0.3 \mathrm{~mL})$ in DCM $(1 \mathrm{~mL})$ at room temperature followed by concentration under reduced pressure to afford a residue which could be triturated and / or recrystallised from hexane/EtOAc mixtures to afford the crystalline acids.

(E)-3-(2-benzylphenyl)acrylic acid (15a): Isolated in 65\% yield as a colourless solid: m.p. (hexanes) 126 - 128 ${ }^{\circ} \mathrm{C} ;{ }^{1} \mathrm{H}$ NMR $(\mathrm{DMSO}, 360 \mathrm{MHz}) \delta 7.89(\mathrm{~d}, 1 \mathrm{H}, \mathrm{J}=15.8), 7.77(\mathrm{~d}, 1 \mathrm{H}, \mathrm{J}=7.6), 7.43-7.34(\mathrm{~m}, 5 \mathrm{H}), 7.19(\mathrm{~m}$, 
$1 \mathrm{H}), 7.11(\mathrm{~d}, 2 \mathrm{H}, \mathrm{J}=7.3) 6.41(\mathrm{~d}, 1 \mathrm{H}, \mathrm{J}=15.8), 4.15(\mathrm{~s}, 2 \mathrm{H}) ;{ }^{13} \mathrm{C}$ NMR (DMSO, $\left.90.55 \mathrm{MHz}\right) \delta 166.9$ (quat), 140.7(quat), $139.9(\mathrm{CH}), 139.5(\mathrm{CH}), 132.4$ (quat), $130.4(\mathrm{CH}), 129.7$ (quat), $128.0(\mathrm{CH}), 127.8(\mathrm{CH}), 126.5$ $(\mathrm{CH}), 126.3(\mathrm{CH}), 125.5(\mathrm{CH}), 119.8(\mathrm{CH}), 37.6\left(\mathrm{CH}_{2}\right)$; IR (film/ $\left.\mathrm{cm}^{-1}\right)$ 1684, 1624, 1418, 1283, 1220; HRMS $\left(\mathrm{ES}^{-}\right)$cald for $\mathrm{C}_{16} \mathrm{H}_{13} \mathrm{O}_{2}:(\mathrm{M}-\mathrm{H})^{-}$237.0910. Found: 237.0911.

(E)-tert-butyl 3-(2-(4-methoxybenzyl)phenyl)acrylate (15b): Isolated in a 76\% yield as a colourless oil; ${ }^{1} \mathrm{H}$ NMR $\left(\mathrm{CDCl}_{3}, 360 \mathrm{MHz}\right) \delta 7.98(\mathrm{~d}, 1 \mathrm{H}, \mathrm{J}=15.8), 7.62(\mathrm{dd}, 1 \mathrm{H}, \mathrm{J}=7.4,1.1), 7.45-7.22(\mathrm{~m}, 3 \mathrm{H}), 7.11(\mathrm{~d}, 2 \mathrm{H}, \mathrm{J}$ $=8.6), 6.87(\mathrm{~d}, 2 \mathrm{H}, \mathrm{J}=8.6), 6.30(\mathrm{~d}, 1 \mathrm{H}, \mathrm{J}=15.8), 4.12(\mathrm{~s}, 2 \mathrm{H}), 3.82(\mathrm{~s}, 3 \mathrm{H}), 1.58(\mathrm{~s}, 9 \mathrm{H}) ;{ }^{13} \mathrm{C} \mathrm{NMR}\left(\mathrm{CDCl}_{3}\right.$, $90.55 \mathrm{MHz}) \delta 165.9$ (quat), 157.7 (quat), 140.9 (quat), $140.2(\mathrm{CH}), 133.4$ (quat), 132.0 (quat), $130.2(\mathrm{CH})$, 129.6(CH), $129.4(\mathrm{CH}), 126.5(\mathrm{CH}), 126.4(\mathrm{CH}), 121.4(\mathrm{CH}), 113.6(\mathrm{CH}) 80.1$ (quat), $54.9\left(\mathrm{CH}_{3}\right), 37.9\left(\mathrm{CH}_{2}\right)$, $27.9\left(\mathrm{CH}_{3}\right)$; IR (film/ $\left.\mathrm{cm}^{-1}\right)$ 2977, 1707, 1511, 1320, 1248, 1150; HRMS (ES ) cald for $\mathrm{C}_{21} \mathrm{H}_{25} \mathrm{O}_{3}$ : $(\mathrm{M}+\mathrm{H})^{+}$ 325.1798. Found: 325.1802 .

(E)-tert-butyl 3-(2-(4-nitrobenzyl)phenyl)acrylate (15c): Isolated in 69\% yield as a colourless oil; ${ }^{1} \mathrm{H}$ NMR $\left(\mathrm{CDCl}_{3}, 360 \mathrm{MHz}\right) \delta 8.17(\mathrm{~d}, 2 \mathrm{H}, \mathrm{J}=8.4), 7.82(\mathrm{~d}, 1 \mathrm{H}, \mathrm{J}=15.8), 7.63(\mathrm{dd}, 1 \mathrm{H}, \mathrm{J}=7.4,1.4), 7.42-7.34(\mathrm{~m}, 2 \mathrm{H})$, $7.32(\mathrm{~d}, 2 \mathrm{H}, \mathrm{J}=8.4), 7.23(\mathrm{dd}, 1 \mathrm{H}, \mathrm{J}=7.4,1.4), 6.29(\mathrm{~d}, 1 \mathrm{H}, \mathrm{J}=15.8), 4.26(\mathrm{~s}, 2 \mathrm{H}), 1.50(\mathrm{~s}, 9 \mathrm{H}) ;{ }^{13} \mathrm{C} \mathrm{NMR}$ $\left(\mathrm{CDCl}_{3}, 90.55 \mathrm{MHz}\right) \delta 165.7$ (quat), 147.8 (quat), 146.2 (quat), $140.1(\mathrm{CH}), 137.8$ (quat), 133.6 (quat), 130.6 $(\mathrm{CH}), 129.9(\mathrm{CH}), 129.2(\mathrm{CH}), 127.4(\mathrm{CH}), 126.8(\mathrm{CH}), 123.6(\mathrm{CH}), 122.1(\mathrm{CH}), 80.5$ (quat), $38.7\left(\mathrm{CH}_{2}\right), 27.9$ $\left(\mathrm{CH}_{3}\right)$; IR (film/ $\left.\mathrm{cm}^{-1}\right)$ 2977, 1707, 1519, 1346, 1151; HRMS $\left(\mathrm{ES}^{+}\right)$cald for $\mathrm{C}_{16} \mathrm{H}_{12} \mathrm{NO}_{3}$ : $\left(\mathrm{M}-\mathrm{O}^{t} \mathrm{Bu}\right)^{+} 266.0812$. Found: 266.0815.

(E)-3-(2-(4-bromobenzyl)phenyl)acrylic acid (15d): Isolated in a yield of 80\% as a light yellow solid; m.p. (hexanes) $174{ }^{\circ} \mathrm{C} ;{ }^{1} \mathrm{H}$ NMR (DMSO, $\left.360 \mathrm{MHz}\right) \delta 7.99(\mathrm{~d}, 1 \mathrm{H}, \mathrm{J}=15.8), 7.69(\mathrm{~d}, 1 \mathrm{H}, \mathrm{J}=6.4,1.4), 7.48(\mathrm{~d}, 2 \mathrm{H}, \mathrm{J}$ $=8.3), 7.43-7.31(\mathrm{~m}, 3 \mathrm{H}), 7.08(\mathrm{~d}, 2 \mathrm{H}, \mathrm{J}=8.3), 6.41(\mathrm{~d}, 1 \mathrm{H}, \mathrm{J}=15.8), 4.14(\mathrm{~s}, 2 \mathrm{H}) ;{ }^{13} \mathrm{C}$ NMR $(\mathrm{DMSO}, 90.55$ MHz) $\delta 167.3$ (quat), $141.0(\mathrm{CH}), 139.9$ (quat), 139.4 (quat), 132.9 (quat), $131.3(\mathrm{CH}), 130.9(\mathrm{CH}), 130.6(\mathrm{CH})$, $130.2(\mathrm{CH}), 127.2(\mathrm{CH}), 126.9(\mathrm{CH}), 120.6(\mathrm{CH}), 119.1(\mathrm{CH}), 37.4\left(\mathrm{CH}_{2}\right)$; IR $\left(\mathrm{film} / \mathrm{cm}^{-1}\right)$ 1685, 1627, 1486, 1418, 1281, 1217; HRMS (ES) cald for $\mathrm{C}_{16} \mathrm{H}_{12} \mathrm{O}_{2} \mathrm{Br}$ : $(\mathrm{M}-\mathrm{H})^{-}$315.0015. Found: 315.0022 .

(E)-3-(2-(4-fluorobenzyl)phenyl)acrylic acid (15e): Isolated as a colourless solid in a 69\% yield. m.p (hexanes) $147{ }^{\circ} \mathrm{C} ;{ }^{1} \mathrm{H}$ NMR (DMSO, $\left.360 \mathrm{MHz}\right) \delta 7.86(\mathrm{~d}, 1 \mathrm{H}, \mathrm{J}=15.8), 7.76(\mathrm{~d}, 1 \mathrm{H}, \mathrm{J}=7.9), 7.41(\mathrm{~m}, 1 \mathrm{H}), 7.33(\mathrm{t}, 2 \mathrm{H}, \mathrm{J}=$ 7.3), 7.18 - $7.09(\mathrm{~m}, 4 \mathrm{H}), 6.41(\mathrm{~d}, 1 \mathrm{H}, \mathrm{J}=15.8), 4.16(\mathrm{~s}, 2 \mathrm{H}) ;{ }^{13} \mathrm{C}$ NMR (DMSO, $\left.90.55 \mathrm{MHz}\right) \delta 167.3$ (quat), 161.9, 159.3 (CF), 141.0(CH), 139.8(quat), 136.6 (quat), 132.9 (quat), $130.8(\mathrm{CH}), 130.2(\mathrm{CH}), 130.1(\mathrm{CH})$, $130.0(\mathrm{CH}), 127.1(\mathrm{CH}), 126.9(\mathrm{CH}), 120.5(\mathrm{CH}), 115.3(\mathrm{CH}), 115.1(\mathrm{CH}), 37.2\left(\mathrm{CH}_{2}\right) ; \mathrm{IR}\left(\mathrm{film} / \mathrm{cm}^{-1}\right)$ 1680, 1621, 1509, 1224; HRMS (ES-) cald for $\mathrm{C}_{16} \mathrm{H}_{12} \mathrm{O}_{2} \mathrm{~F}:(\mathrm{M}-\mathrm{H})^{-}$255.0816. Found: 255.0809.

(E)-3-(2-(3-chlorobenzyl)phenyl)acrylic acid (15f): Isolated as a white powdery solid in a yield of 91\%. m.p. (hexanes) $142{ }^{\circ} \mathrm{C} ;{ }^{1} \mathrm{H}$ NMR (DMSO, $\left.360 \mathrm{MHz}\right) \delta 7.86(\mathrm{~d}, 1 \mathrm{H}, \mathrm{J}=15.8), 7.77(\mathrm{dd}, 1 \mathrm{H}, \mathrm{J}=8.7,1.7), 7.43(\mathrm{~m}$, $1 \mathrm{H}), 7.36-7.30(\mathrm{~m}, 3 \mathrm{H}), 7.26(\mathrm{~m}, 1 \mathrm{H}), 7.18(\mathrm{t}, 1 \mathrm{H}, \mathrm{J}=1.7), 7.09(\mathrm{bd}, 1 \mathrm{H}, \mathrm{J}=7.6), 6.41(\mathrm{~d}, 1 \mathrm{H}, \mathrm{J}=15.8), 4.18$ $(\mathrm{s}, 2 \mathrm{H}) ;{ }^{13} \mathrm{C}$ NMR (DMSO, $\left.90.55 \mathrm{MHz}\right) \delta 167.2$ (quat), 143.0 (quat), $140.9(\mathrm{CH}), 139.1$ (quat), 133.0 (quat), 132.9 (quat), $130.8(\mathrm{CH}), 130.2(\mathrm{CH}), 128.1(\mathrm{CH}), 127.2(\mathrm{CH}), 127.0(\mathrm{CH}), 126.9(\mathrm{CH}), 126.0(\mathrm{CH}), 120.6$ $(\mathrm{CH}), 37.5\left(\mathrm{CH}_{2}\right)$; IR (film/ $\left.\mathrm{cm}^{-1}\right)$ 1681, 1621, 1416, 1279, 1223; HRMS (ES) cald for $\mathrm{C}_{16} \mathrm{H}_{12} \mathrm{O}_{2} \mathrm{Cl}$ : $(\mathrm{M}-\mathrm{H})^{-}$ 271.0520. Found: 271.0512 .

(E)-3-(2-(4-cyanobenzyl)phenyl)acrylic acid (15g): Isolated as a colourless powdery solid in a yield of 89\%. m.p. (hexanes) $175{ }^{\circ} \mathrm{C} ;{ }^{1} \mathrm{H}$ NMR (DMSO, $\left.360 \mathrm{MHz}\right) \delta 7.95-7.76(\mathrm{~m}, 4 \mathrm{H}), 7.44-7.30(\mathrm{~m}, 5 \mathrm{H}), 6.41(\mathrm{~d}, 1 \mathrm{H}, \mathrm{J}=$ 15.8), 4.27 (s, 2H); ${ }^{13} \mathrm{C}$ NMR (DMSO, $90.55 \mathrm{MHz}$ ) $\delta 168.7$ (quat), 148.0 (quat), 142.3 (CH), 140.2 (quat), 134.5 (quat), $133.9(\mathrm{CH}), 132.5(\mathrm{CH}), 131.8(\mathrm{CH}), 128.9(\mathrm{CH}), 128.5(\mathrm{CH}), 122.2(\mathrm{CH}), 120.3$ (quat), 110.4 (quat), $39.5\left(\mathrm{CH}_{2}\right)$; IR (film/cm $\left.{ }^{-1}\right)$ 2360, 2341, 1677, 1623; HRMS (ES') cald for $\mathrm{C}_{17} \mathrm{H}_{12} \mathrm{NO}_{2}$ : $(\mathrm{M}-\mathrm{H})^{-} 262.0862$. Found: 262.0867 .

(E)-3-(2-(2-nitrobenzyl)phenyl)acrylic acid (15h): Isolated as a powdery yellow solid in a yield of 58\%. m.p. (hexanes) $157{ }^{\circ} \mathrm{C} ;{ }^{1} \mathrm{H}$ NMR (DMSO, $\left.360 \mathrm{MHz}\right) \delta 8.05(\mathrm{~d}, 1 \mathrm{H}, \mathrm{J}=7.5), 7.82-7.78(\mathrm{~m}, 2 \mathrm{H}), 7.67(\mathrm{t}, 1 \mathrm{H}, \mathrm{J}=6.9)$, 
$7.55(\mathrm{t}, 1 \mathrm{H}, \mathrm{J}=7.5), 7.36-7.31(\mathrm{~m}, 2 \mathrm{H}), 7.21(\mathrm{~d}, 1 \mathrm{H}, \mathrm{J}=7.6), 7.00(\mathrm{~d}, 1 \mathrm{H}, \mathrm{J}=8.6), 6.43(\mathrm{~d}, 1 \mathrm{H}, \mathrm{J}=15.7), 4.46$ $(\mathrm{s}, 2 \mathrm{H}) ;{ }^{13} \mathrm{C}$ NMR (DMSO, 90.55 MHz) $\delta 167.2$ (quat), 148.8 (quat), $140.6(\mathrm{CH}), 137.8$ (quat), 134.3 (quat), $133.6(\mathrm{CH}), 133.2$ (quat), $132.0(\mathrm{CH}), 130.2(\mathrm{CH}), 129.8(\mathrm{CH}), 128.0(\mathrm{CH}), 127.3(\mathrm{CH}), 127.0(\mathrm{CH}), 124.8$ $(\mathrm{CH}), 121.0(\mathrm{CH}), 34.6\left(\mathrm{CH}_{2}\right)$; IR (film/ $\left.\mathrm{cm}^{-1}\right)$ 1699, 1634, 1523, 1348; HRMS (ES) cald for $\mathrm{C}_{16} \mathrm{H}_{12} \mathrm{NO}_{4}:(\mathrm{M}-\mathrm{H})^{-}$ 282.0761. Found: 282.0763.

(E)-3-(2-(4-(trifluoromethyl)benzyl)phenyl)acrylic acid (15i): Isolated as a colourless crystalline solid in a yield of 67\%; m.p (water) $122-123{ }^{\circ} \mathrm{C} ;{ }^{1} \mathrm{H}$ NMR (DMSO, $\left.360 \mathrm{MHz}\right) \delta 7.85(\mathrm{~d}, 1 \mathrm{H}, \mathrm{J}=15.8), 7.79(\mathrm{~d}, 1 \mathrm{H}, \mathrm{J}=$ 7.3), $7.67(\mathrm{~d}, 2 \mathrm{H}, \mathrm{J}=8.1), 7.42(\mathrm{q}, 1 \mathrm{H}, \mathrm{J}=7.2), 7.36-7.33(\mathrm{~m}, 4 \mathrm{H}), 6.42(\mathrm{~d}, 1 \mathrm{H}, \mathrm{J}=15.8), 4.27(\mathrm{~s}, 2 \mathrm{H}) ;{ }^{13} \mathrm{C}$ NMR (DMSO, $90.55 \mathrm{MHz}$ ) $\delta 167.2$ (quat), 145.4 (quat), $140.9(\mathrm{CH}), 139.0$ (quat), 133.0 (quat), $131.0(\mathrm{CH})$, $130.3(\mathrm{CH}), 129.1(\mathrm{CH}), 127.3(\mathrm{CH}), 127.0(\mathrm{CH}), 127.6\left(\mathrm{CF}_{3}\right) 125.3(\mathrm{CH}), 120.6(\mathrm{CH}), 37.8\left(\mathrm{CH}_{2}\right) ;{ }^{19} \mathrm{~F}$ NMR $(\mathrm{DMSO}, 235 \mathrm{MHz}) \delta-62.0$; IR $\left(\right.$ film $\left./ \mathrm{cm}^{-1}\right)$ 1699, 1633, 1325, 1164, 1122, 1066; HRMS (ES) cald for $\mathrm{C}_{17} \mathrm{H}_{12} \mathrm{O}_{2} \mathrm{~F}_{3}$ : (M-H)- 305.0784. Found: 305.0798.

(E)-3-(2-(3-methoxybenzyl)phenyl)acrylic acid (15j): Isolated as a colourless crystalline solid in a yield of $62 \%$. m.p (water) $134-135^{\circ} \mathrm{C} ;{ }^{1} \mathrm{H}$ NMR (DMSO, $\left.360 \mathrm{MHz}\right) \delta 7.91(\mathrm{~d}, 1 \mathrm{H}, \mathrm{J}=15.8), 7.76(\mathrm{~d}, 1 \mathrm{H}, \mathrm{J}=7.7), 7.40$ $(\mathrm{q}, 1 \mathrm{H}, \mathrm{J}=8.3), 7.33-7.30(\mathrm{~m}, 2 \mathrm{H}), 7.20(\mathrm{t}, 1 \mathrm{H}, \mathrm{J}=7.7), 7.78-6.67(\mathrm{~m}, 3 \mathrm{H}), 6.41(\mathrm{~d}, 1 \mathrm{H}, \mathrm{J}=15.8), 4.11(\mathrm{~s}, 2 \mathrm{H})$, $3.71(\mathrm{~s}, 3 \mathrm{H}) ;{ }^{13} \mathrm{C}$ NMR (DMSO, $\left.90.55 \mathrm{MHz}\right) \delta 167.4$ (quat), 159.3 (quat), 142.0 (quat), $141.2(\mathrm{CH}), 139.9$ (quat), 132.9 (quat), $130.9(\mathrm{CH}), 130.2(\mathrm{CH}), 129.5(\mathrm{CH}), 127.1(\mathrm{CH}), 126.8(\mathrm{CH}), 120.6(\mathrm{CH}) 120.3(\mathrm{CH}), 114.2$ $(\mathrm{CH}), 111.3(\mathrm{CH}), 54.8(\mathrm{CH} 3), 38.1(\mathrm{CH} 2)$; IR (film) 1681, 1620, 1279, 1222;. HRMS (ES-) cald for $\mathrm{C}_{17} \mathrm{H}_{15} \mathrm{O}_{3}$ : (M-H)- 267.1016. Found: 267.1016.

(E)-3-(2-((naphthalen-3-yl)methyl)phenyl)acrylic acid (15k): Isolated as a colourless powdery solid in a yield of $92 \%$; m.p. (hexane) $160-161{ }^{\circ} \mathrm{C} ;{ }^{1} \mathrm{H}$ NMR (DMSO, $\left.360 \mathrm{MHz}\right) \delta 7.96(\mathrm{~d}, 1 \mathrm{H}, \mathrm{J}=15.8), 7.88-7.78(\mathrm{~m}, 4 \mathrm{H})$, $7.50(\mathrm{~s}, 1 \mathrm{H}), 7.48-7.32(\mathrm{~m}, 6 \mathrm{H}), 6.41(\mathrm{~d}, 1 \mathrm{H}, \mathrm{J}=15.8), 4.32(\mathrm{~s}, 2 \mathrm{H}) ;{ }^{13} \mathrm{C}$ NMR (DMSO, 90.55 MHz) $\delta 167.4$ (quat), $141.3(\mathrm{CH}), 139.8$ (quat), 138.2 (quat), 133.1 (quat), 133.0 (quat), 131.6 (quat), $131.0(\mathrm{CH}), 130.3(\mathrm{CH})$, $128.0(\mathrm{CH}), 127.5(\mathrm{CH}), 127.4(\mathrm{CH}), 127.2(\mathrm{CH}), 126.9(\mathrm{CH}), 126.4(\mathrm{CH}), 126.2(\mathrm{CH}), 125.6(\mathrm{CH}), 120.4(\mathrm{CH})$, $38.3\left(\mathrm{CH}_{2}\right)$; IR (film/cm $\left.{ }^{-1}\right)$ 2977, 1740, 1707, 1321, 1150; HRMS (ES) cald for $\mathrm{C}_{20} \mathrm{H}_{15} \mathrm{O}_{2}$ : (M-H) 287.1077 . Found: 287.1070.

(E)-tert-butyl 3-(2-(4-methoxybenzyl)-4-methylphenyl)acrylate and (E)-tert-butyl 3-(2-(4-methoxybenzyl)5-methylphenyl)acrylate (15l): Isolated as a colourless oil in a yield of $70 \%$. ${ }^{1} \mathrm{H} \mathrm{NMR}\left(\mathrm{CDCl}_{3}, 360 \mathrm{MHz}\right) \delta$ $7.92(\mathrm{~d}, 1 \mathrm{H}, \mathrm{J}=15.8), 7.50(\mathrm{~d}, 0.5 \mathrm{H}, \mathrm{J}=7.9), 7.41(\mathrm{bs}, 0.5 \mathrm{H}), 7.19-7.12(\mathrm{~m}, 3.5 \mathrm{H}), 7.02(\mathrm{bs}, 0.5 \mathrm{H}), 6.85-$ $6.80(\mathrm{~m}, 2 \mathrm{H}), 6.26(\mathrm{~d}, 0.5 \mathrm{H}, \mathrm{J}=15.8), 6.24(\mathrm{~d}, 0.5 \mathrm{H}, \mathrm{J}=15.8), 4.05(\mathrm{~s}, 2 \mathrm{H}), 3.77(\mathrm{~s}, 3 \mathrm{H}), 1.53(\mathrm{~s}, 9 \mathrm{H}) ;{ }^{13} \mathrm{C} \mathrm{NMR}$ $\left(\mathrm{CDCl}_{3}, 90.55 \mathrm{MHz}\right) \delta 166.3$ (quat), 166.2 (quat), 157.8 (quat), 157.8 (quat), 141.2 (quat), 141.0 (quat), 140.3 $(\mathrm{CH}), 140.0(\mathrm{CH}), 137.5$ (quat), 136.1 (quat), 133.3 (quat), 132.5 (quat), 132.4 (quat), $131.3(\mathrm{CH}), 130.7(\mathrm{CH})$, $130.6(\mathrm{CH}), 130.5(\mathrm{CH}), 129.5(\mathrm{CH}), 127.6(\mathrm{CH}), 127.1(\mathrm{CH}), 126.5(\mathrm{CH}), 121.2(\mathrm{CH}), 120.4(\mathrm{CH}), 113.8(\mathrm{CH})$, 80.2 (quat), 80.2 (quat), $55.1\left(\mathrm{CH}_{3}\right), 38.0\left(\mathrm{CH}_{2}\right), 37.7\left(\mathrm{CH}_{2}\right), 28.1\left(\mathrm{CH}_{3}\right), 21.3\left(\mathrm{CH}_{3}\right), 20.9\left(\mathrm{CH}_{3}\right) ; \mathrm{IR}\left(\mathrm{film} / \mathrm{cm}^{-1}\right)$ 2977, 1707, 1511, 1248, 1150; HRMS $\left(\mathrm{ES}^{+}\right)$cald for $\mathrm{C}_{18} \mathrm{H}_{17} \mathrm{O}_{2}:\left(\mathrm{M}-\mathrm{O}^{\mathrm{t}} \mathrm{Bu}\right)^{+}$265.1223. Found: 265.1213. 
Part B: Spectroscopic Data 

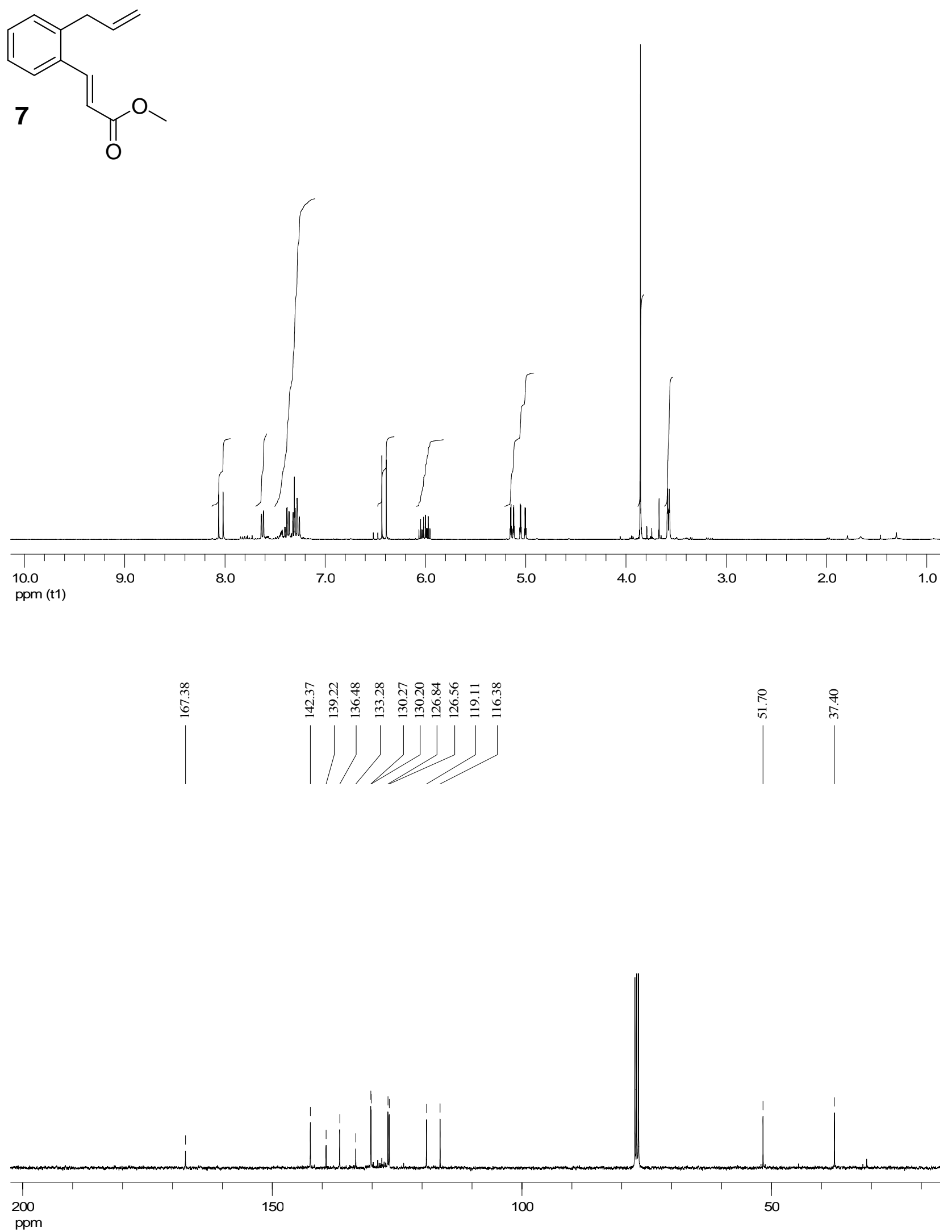

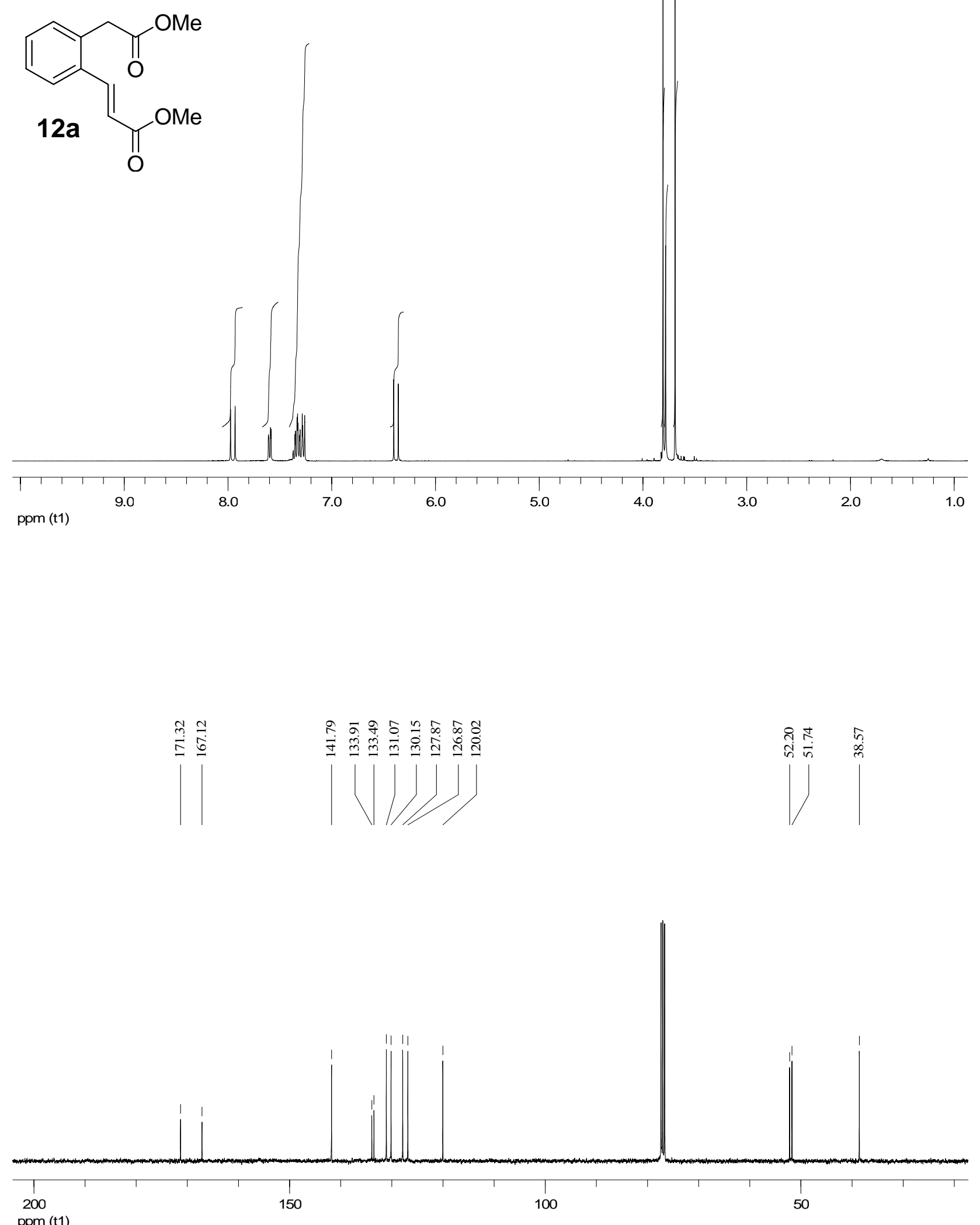


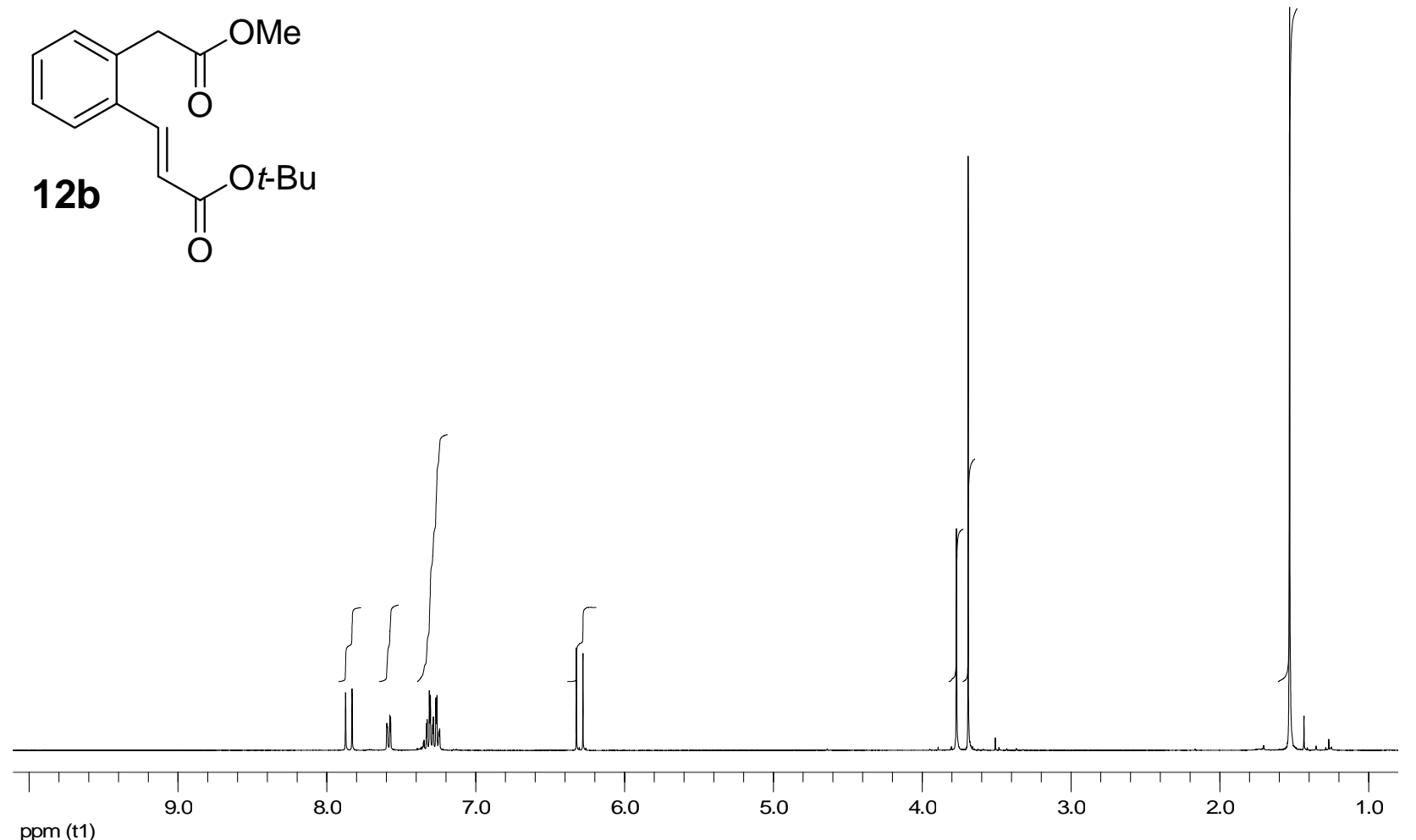

ppm (t1)
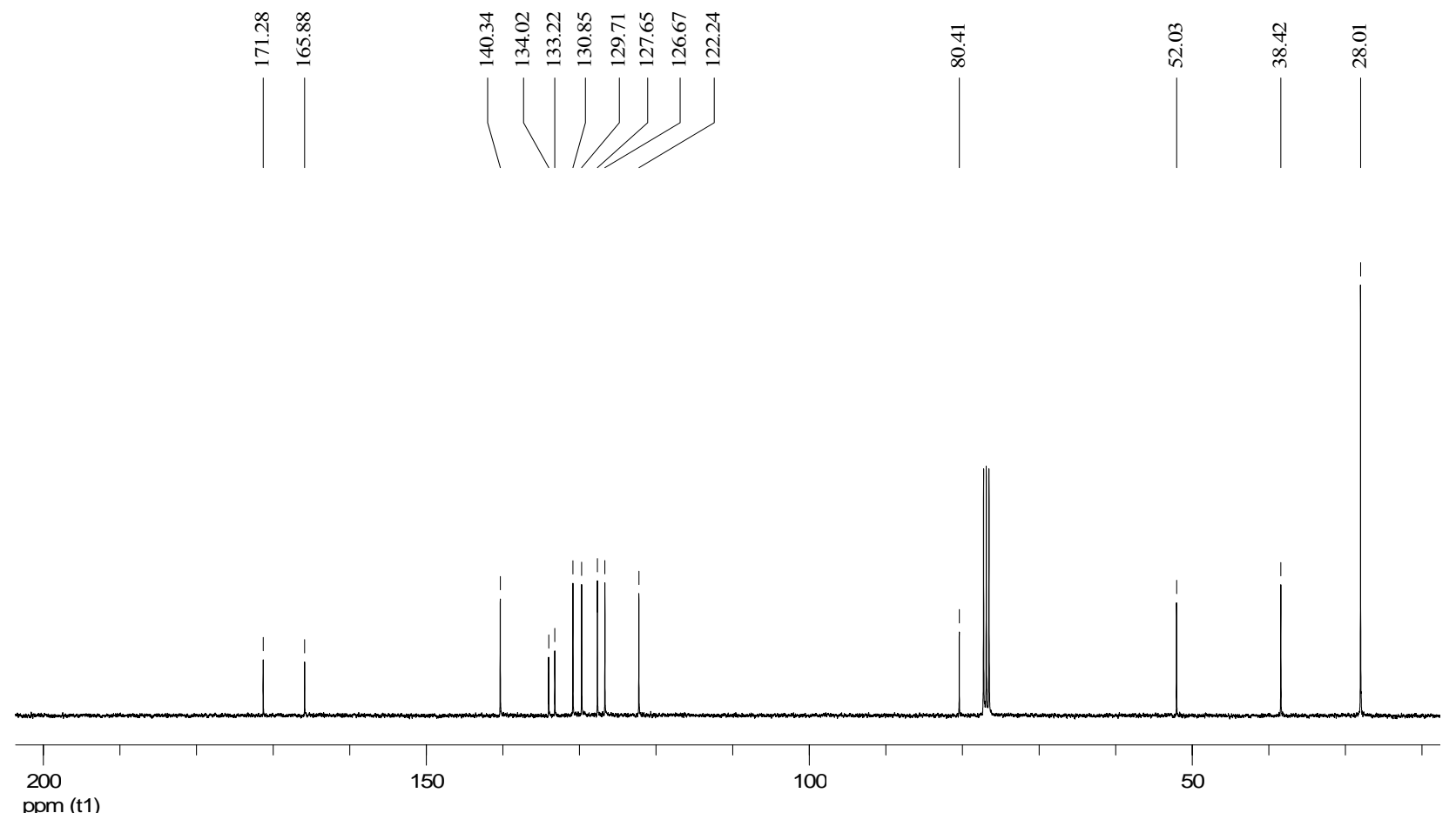


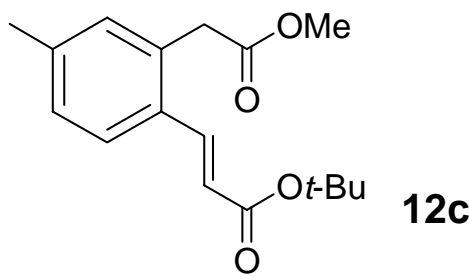<smiles>CCCCOC(=O)/C=C/c1cc(C)ccc1CC(=O)OC</smiles>
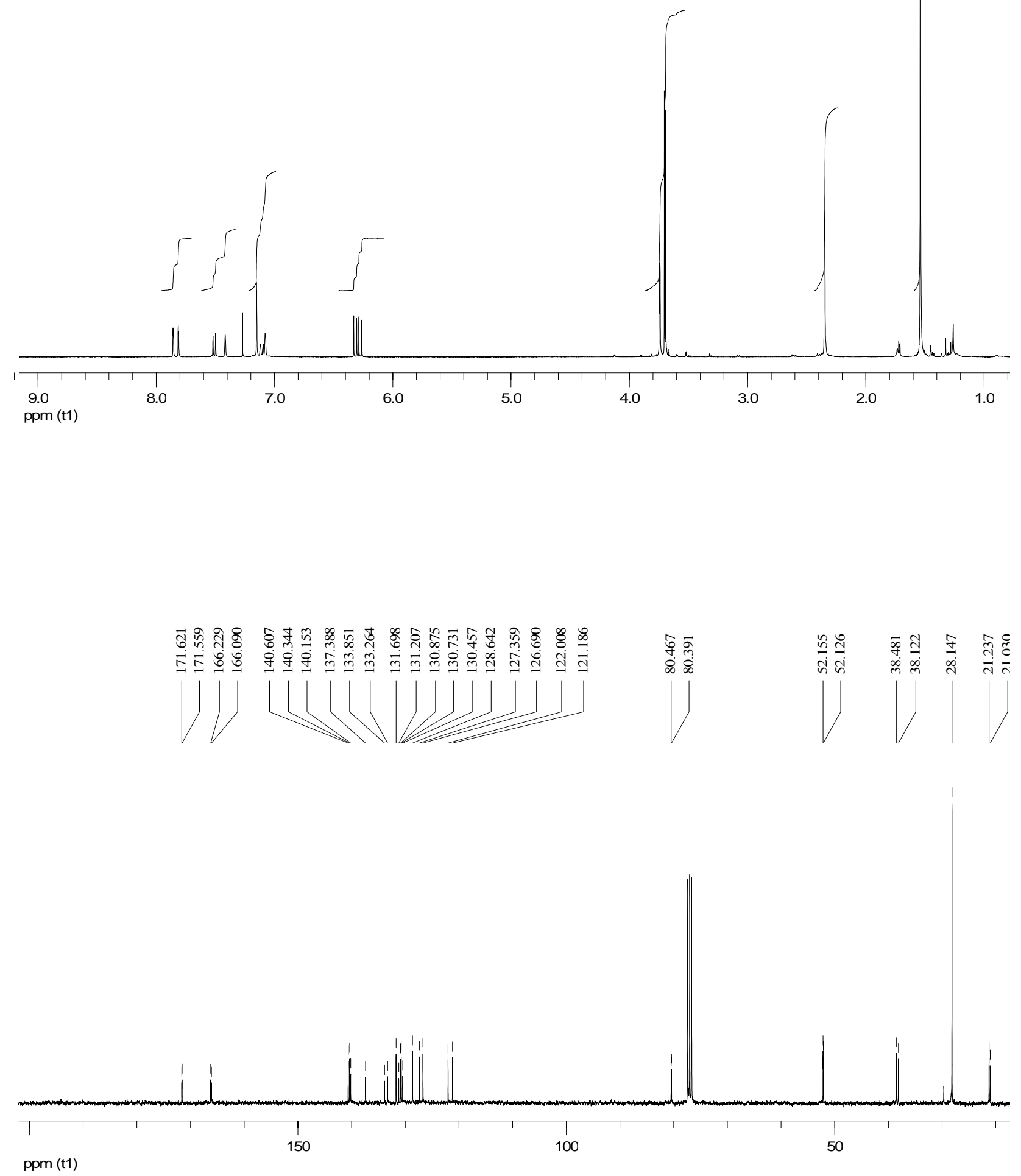

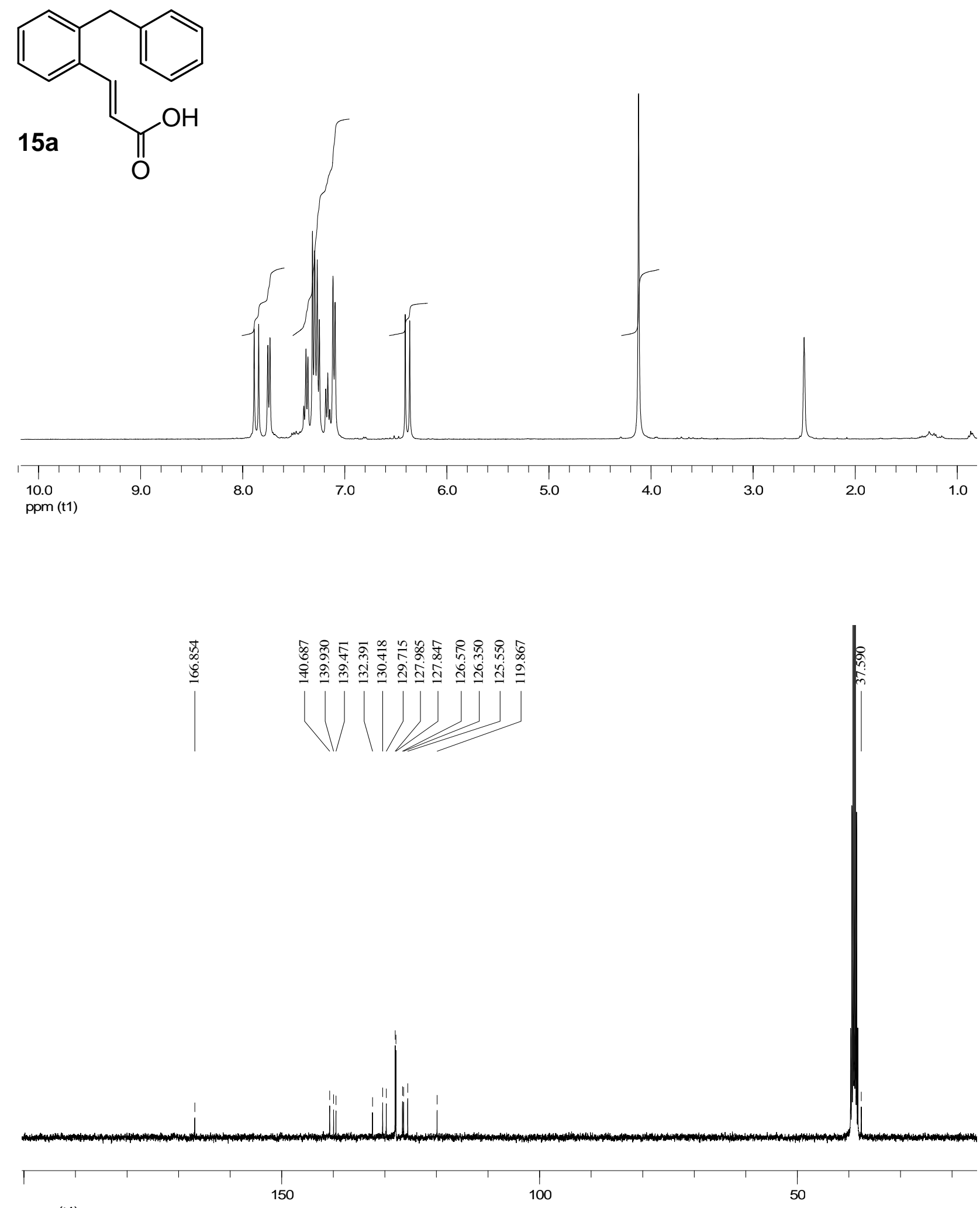

ppm (t1) 
<smiles>CCCCC(=O)/C=C/c1ccccc1Cc1ccc(OC)cc1</smiles>
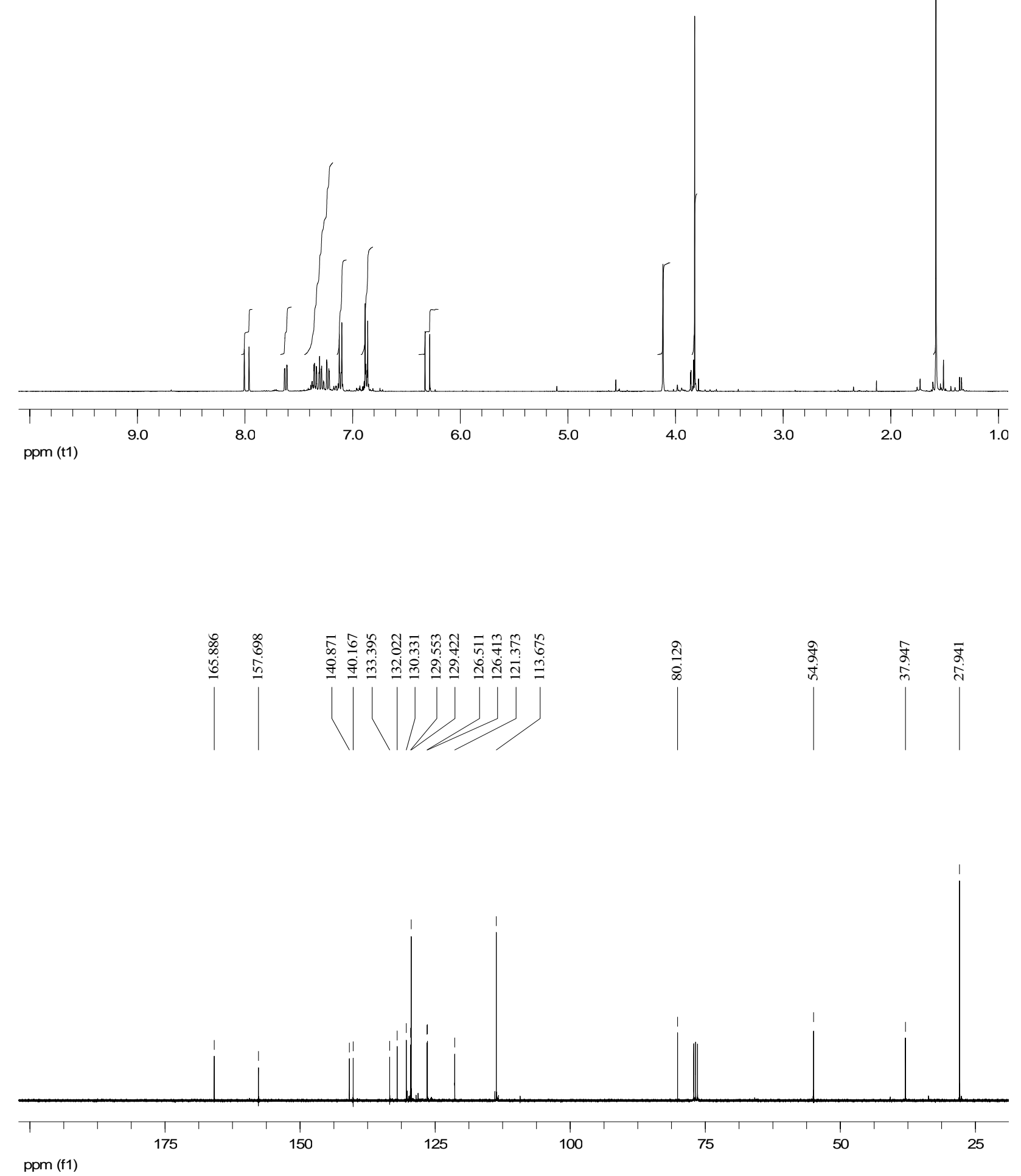


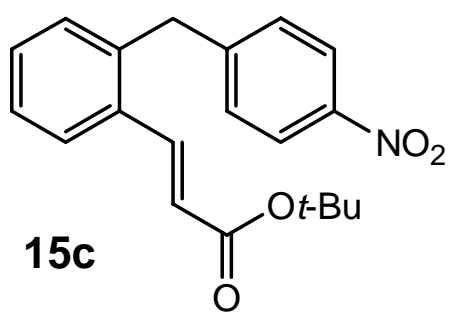

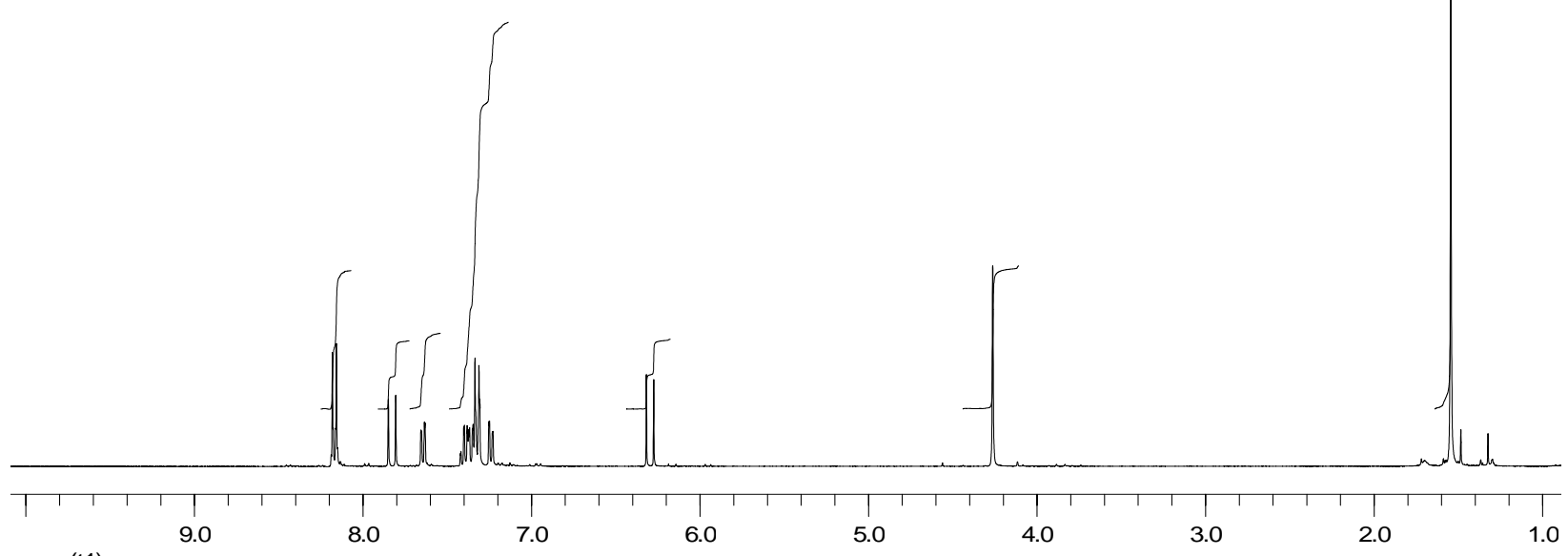

ppm (t1)
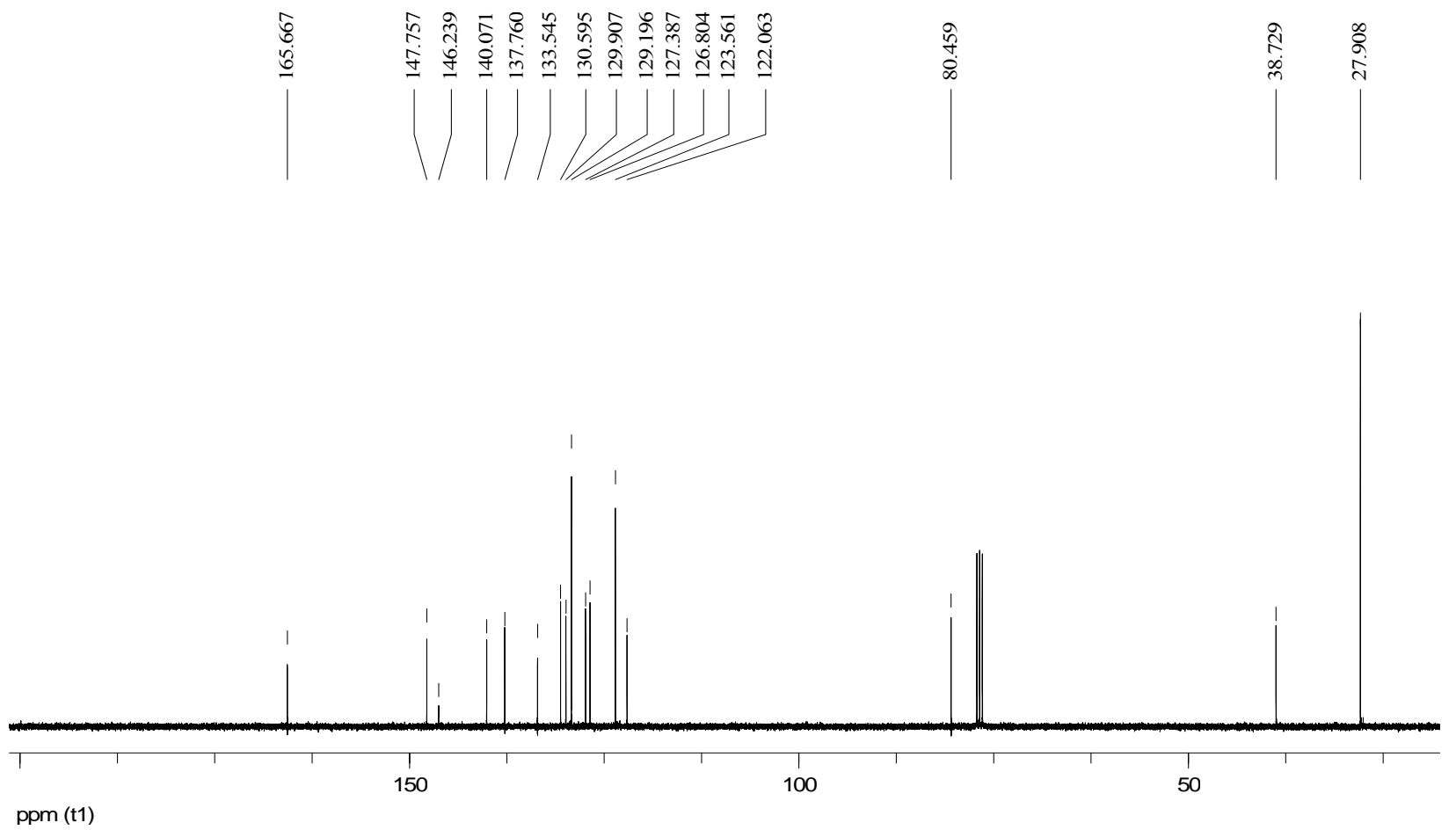

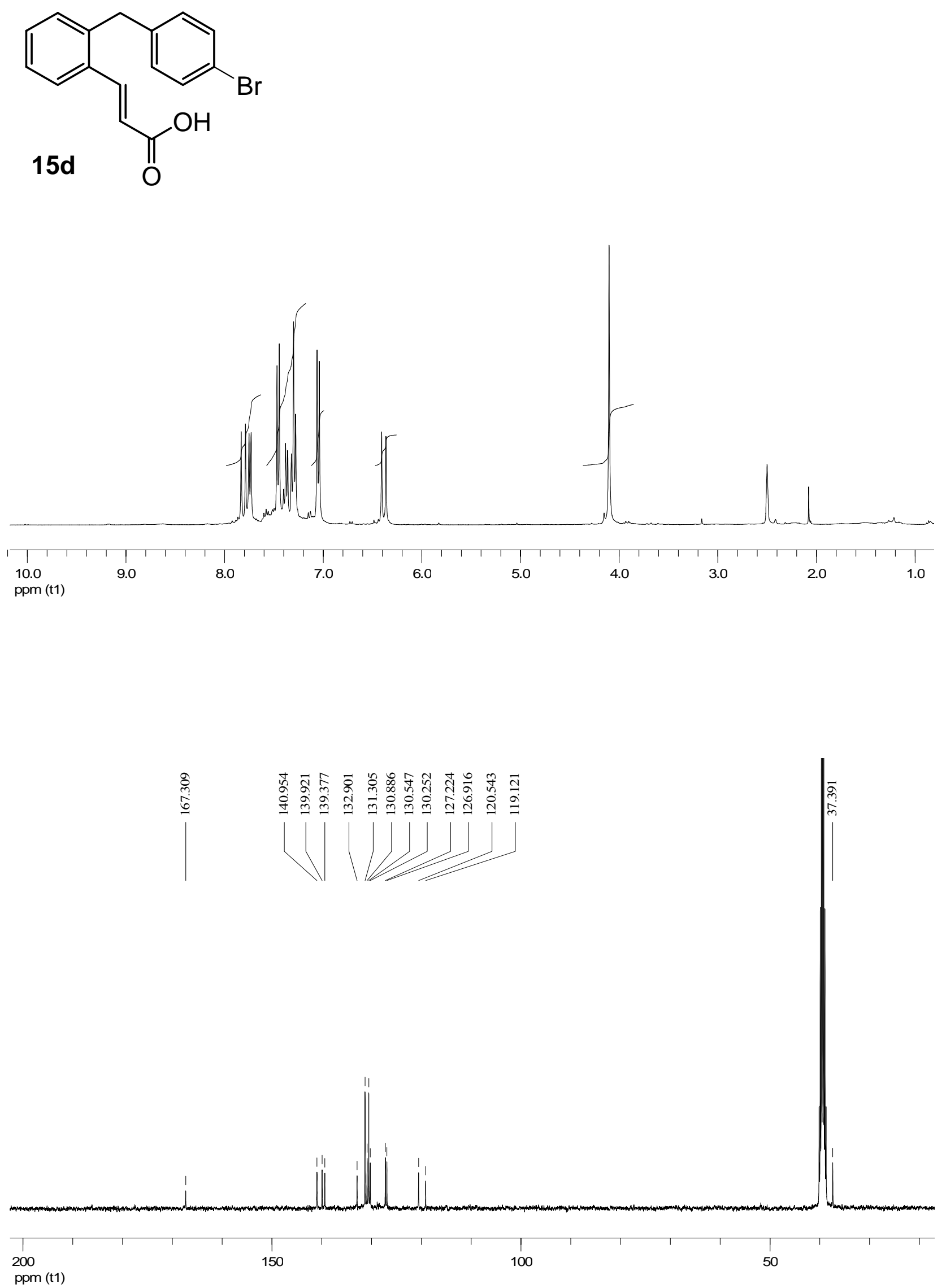
<smiles>O=C(O)/C=C/c1ccccc1Cc1ccc(F)cc1</smiles>

$15 e$

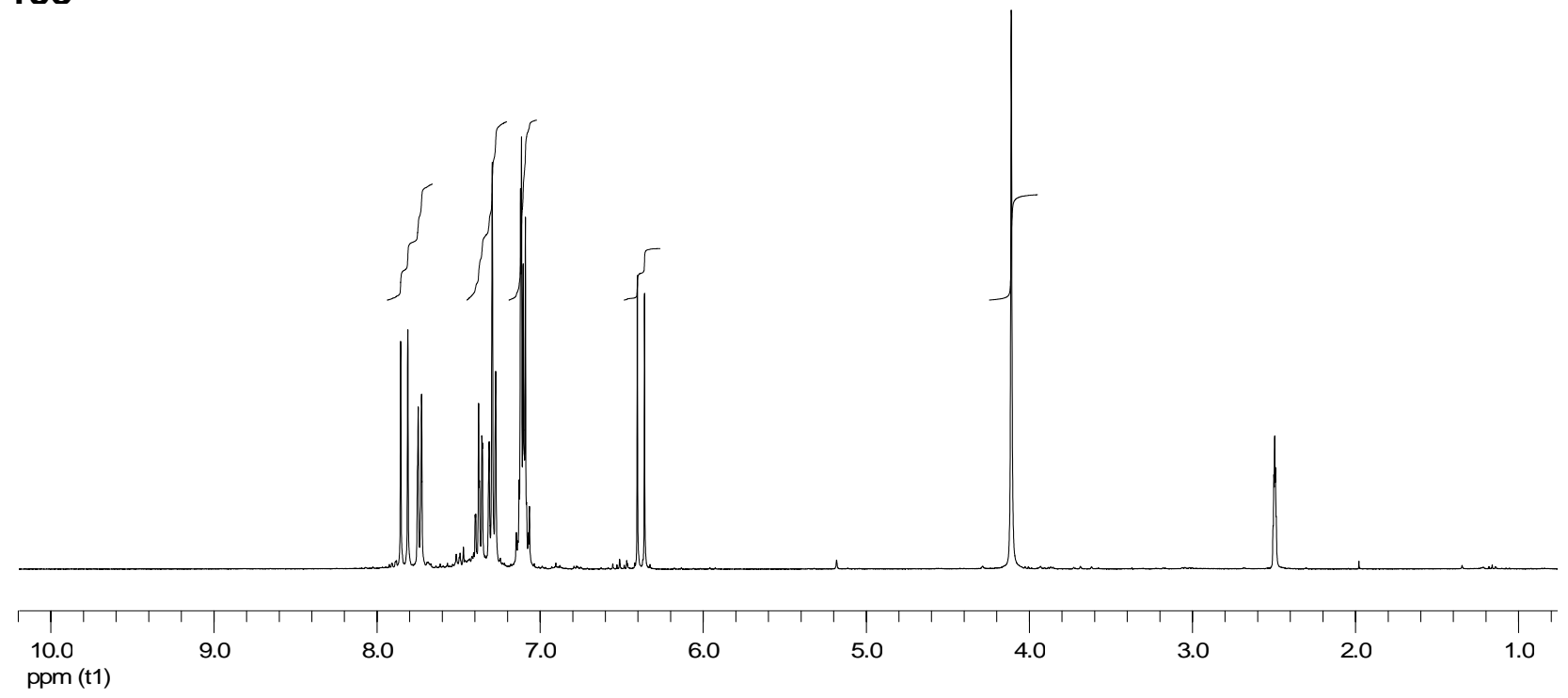

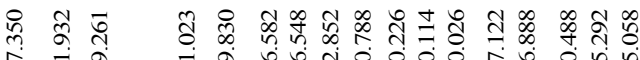

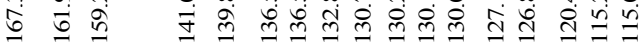
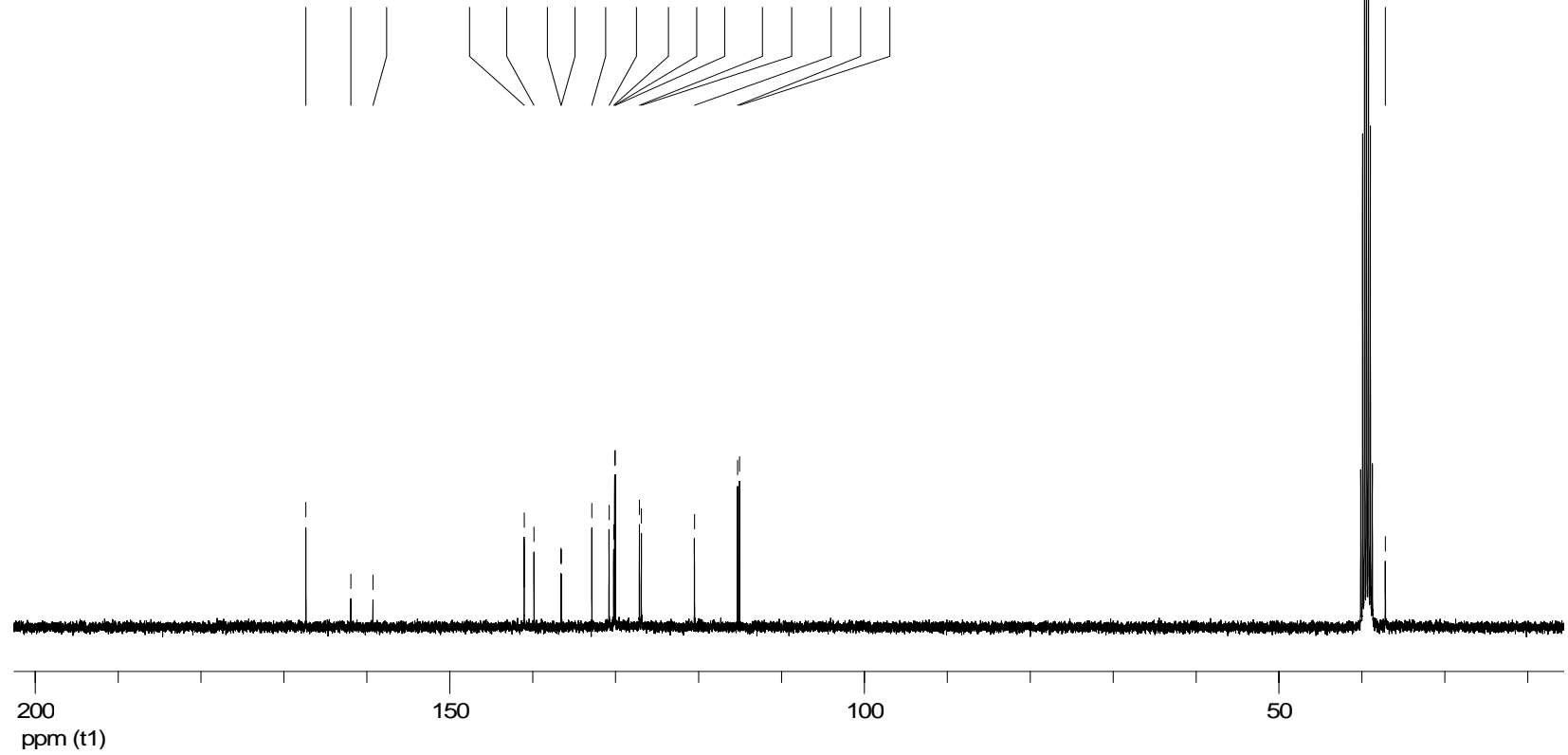
<smiles>[CH-]1C[Te][Te]1</smiles>

$15 f$
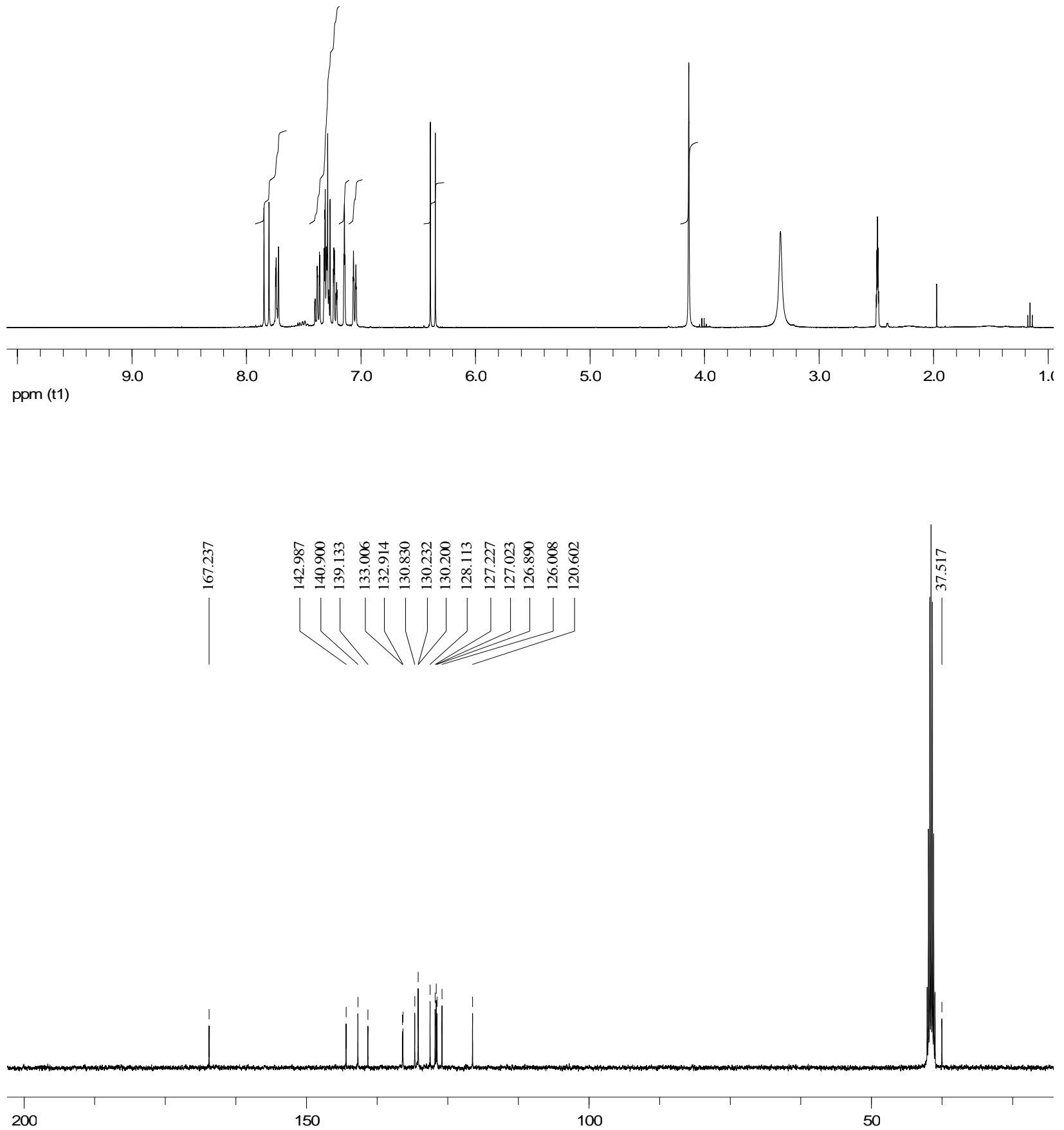

ppm (t1) 
<smiles>CC(C)(C)C=Cc1ccccc1Cc1ccc(C#N)cc1</smiles>
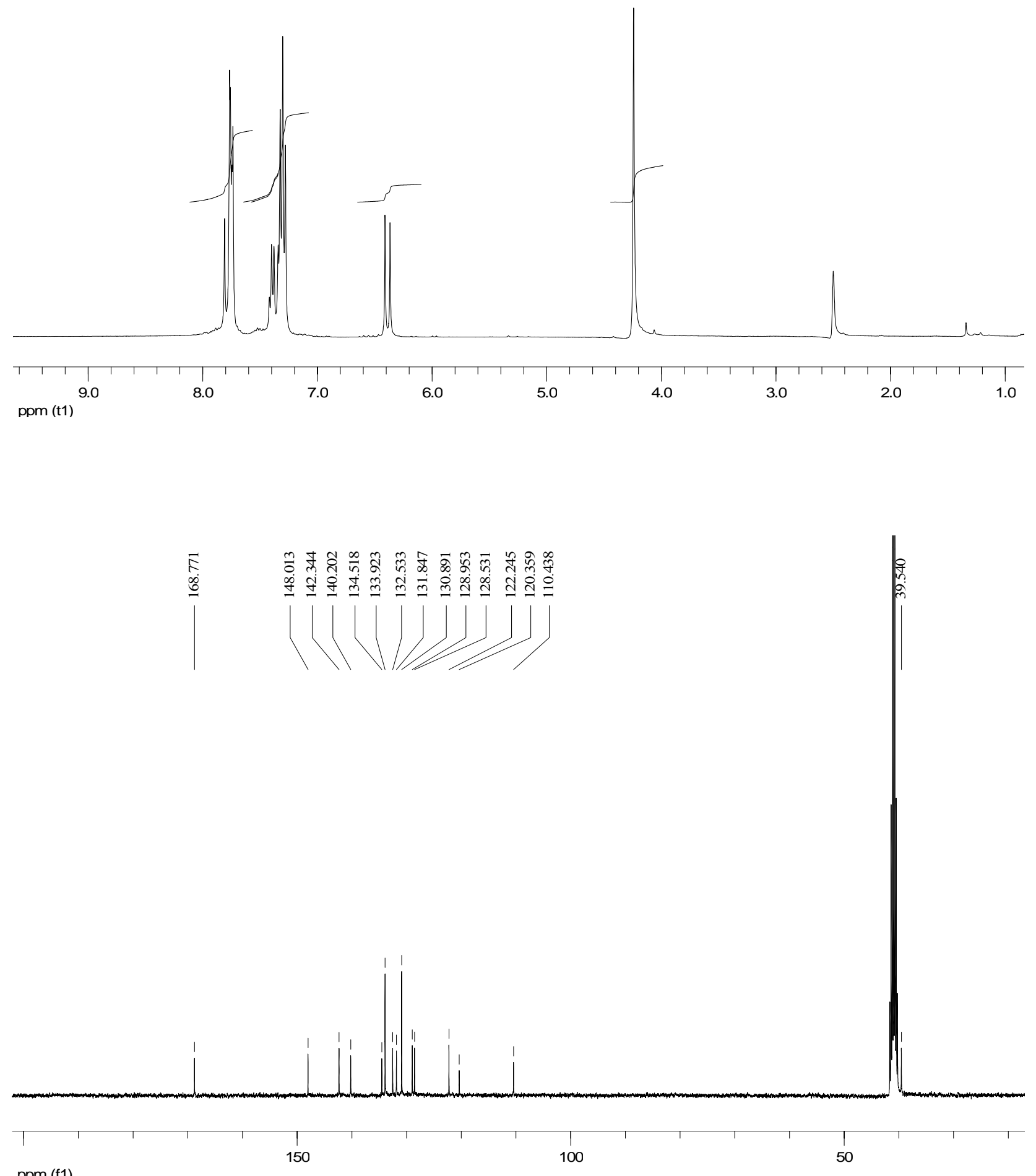

ppm (f1) 

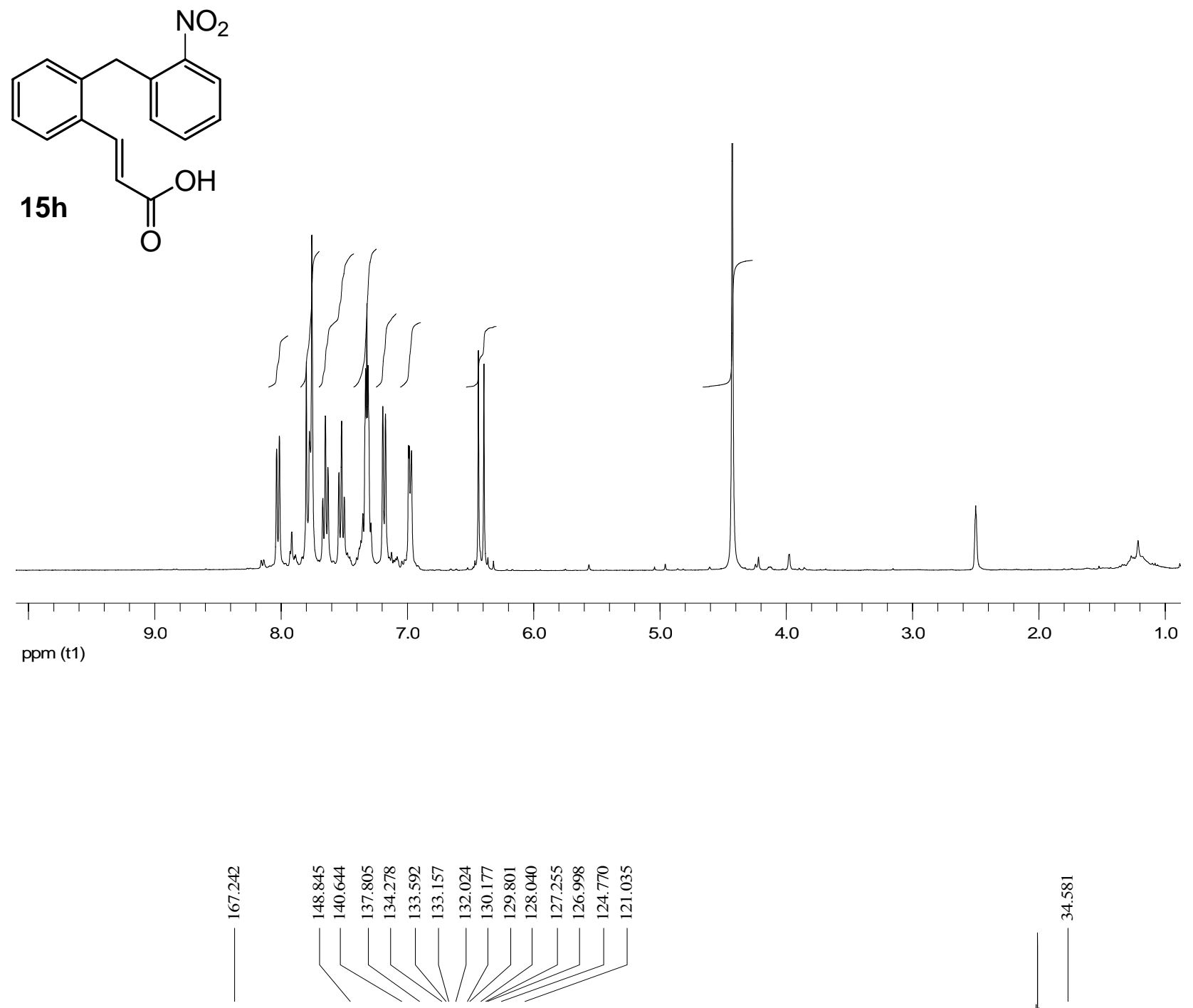

\section{$\vec{\infty}$
$\stackrel{n}{7}$}



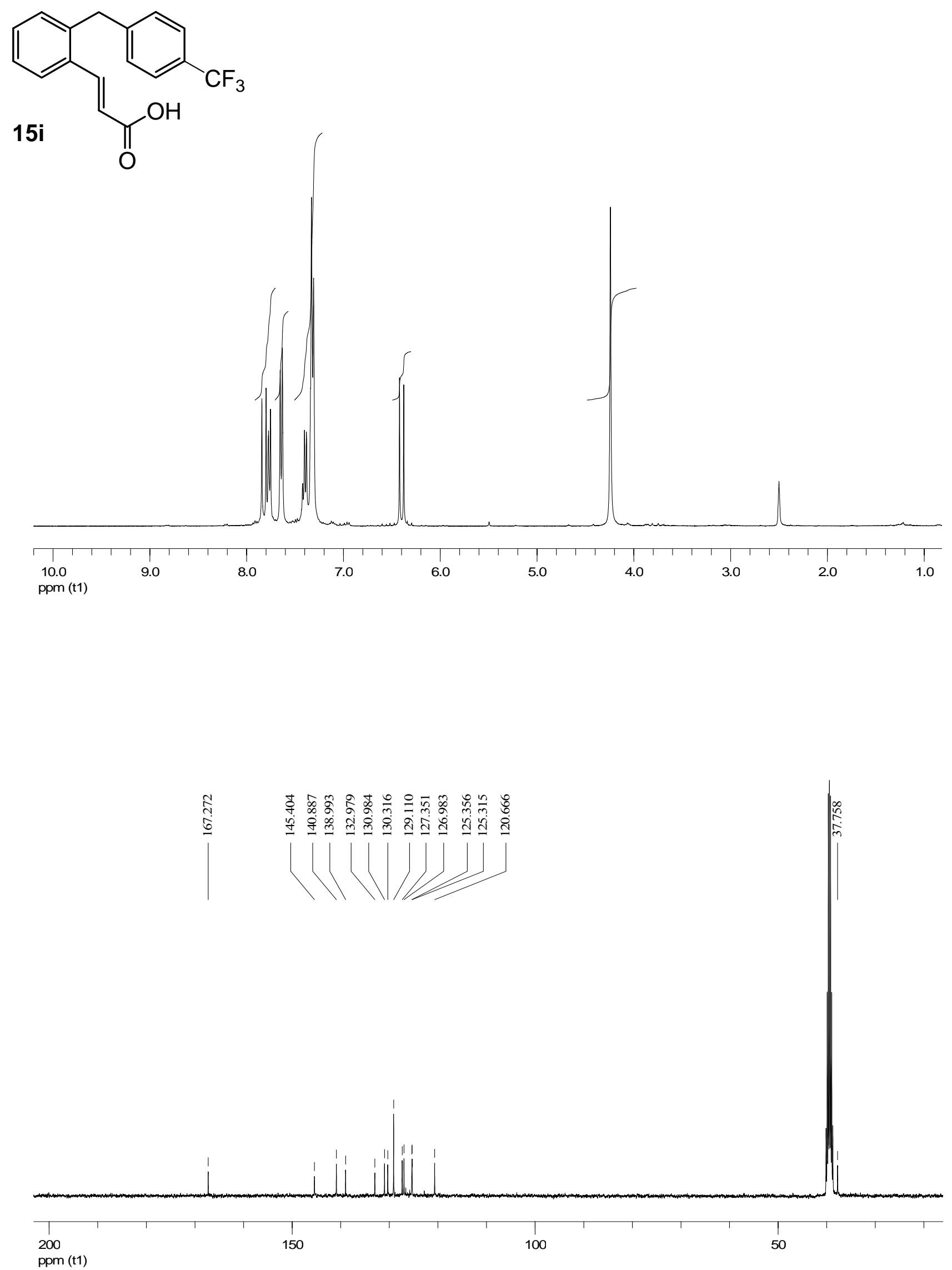

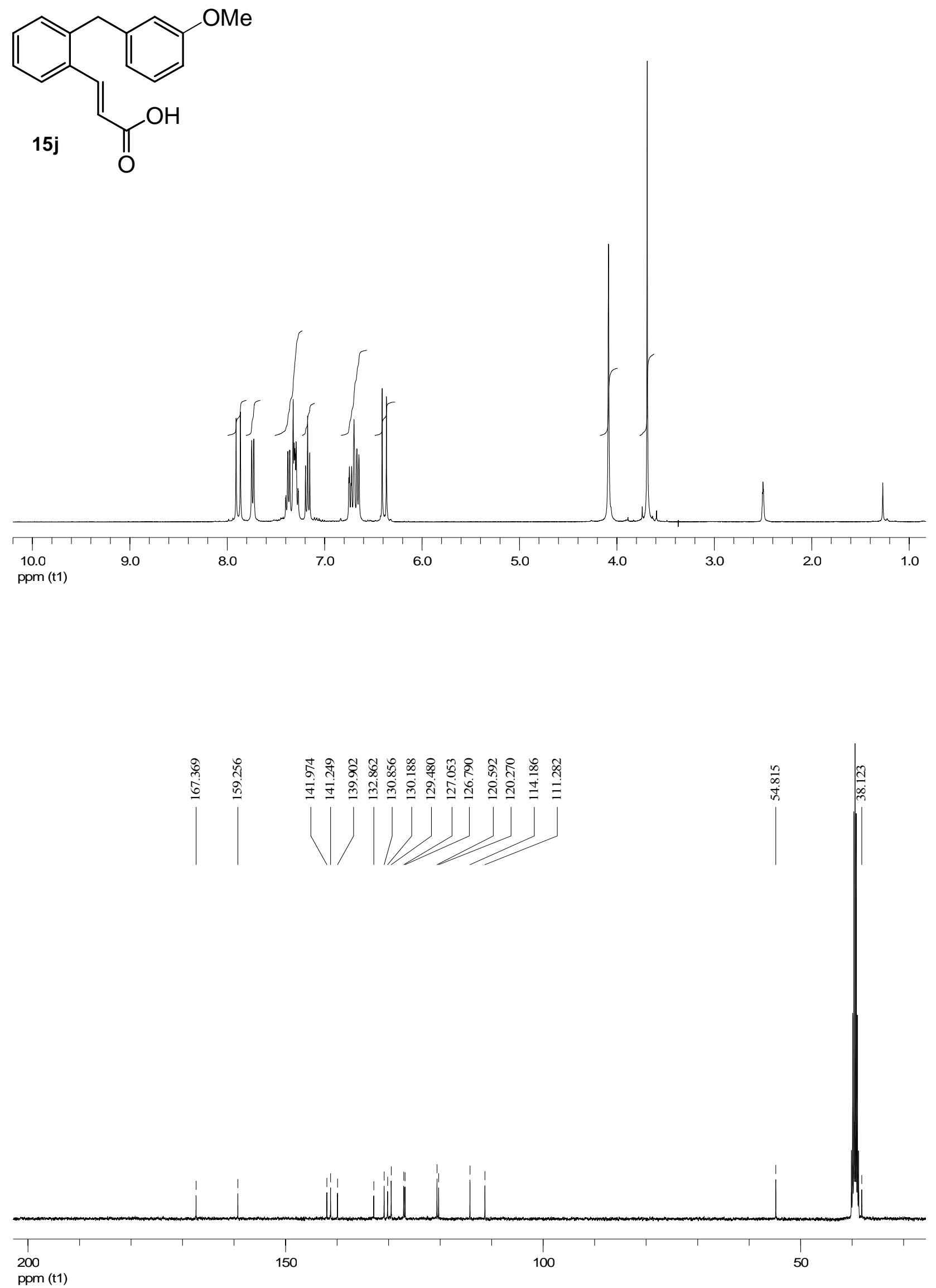


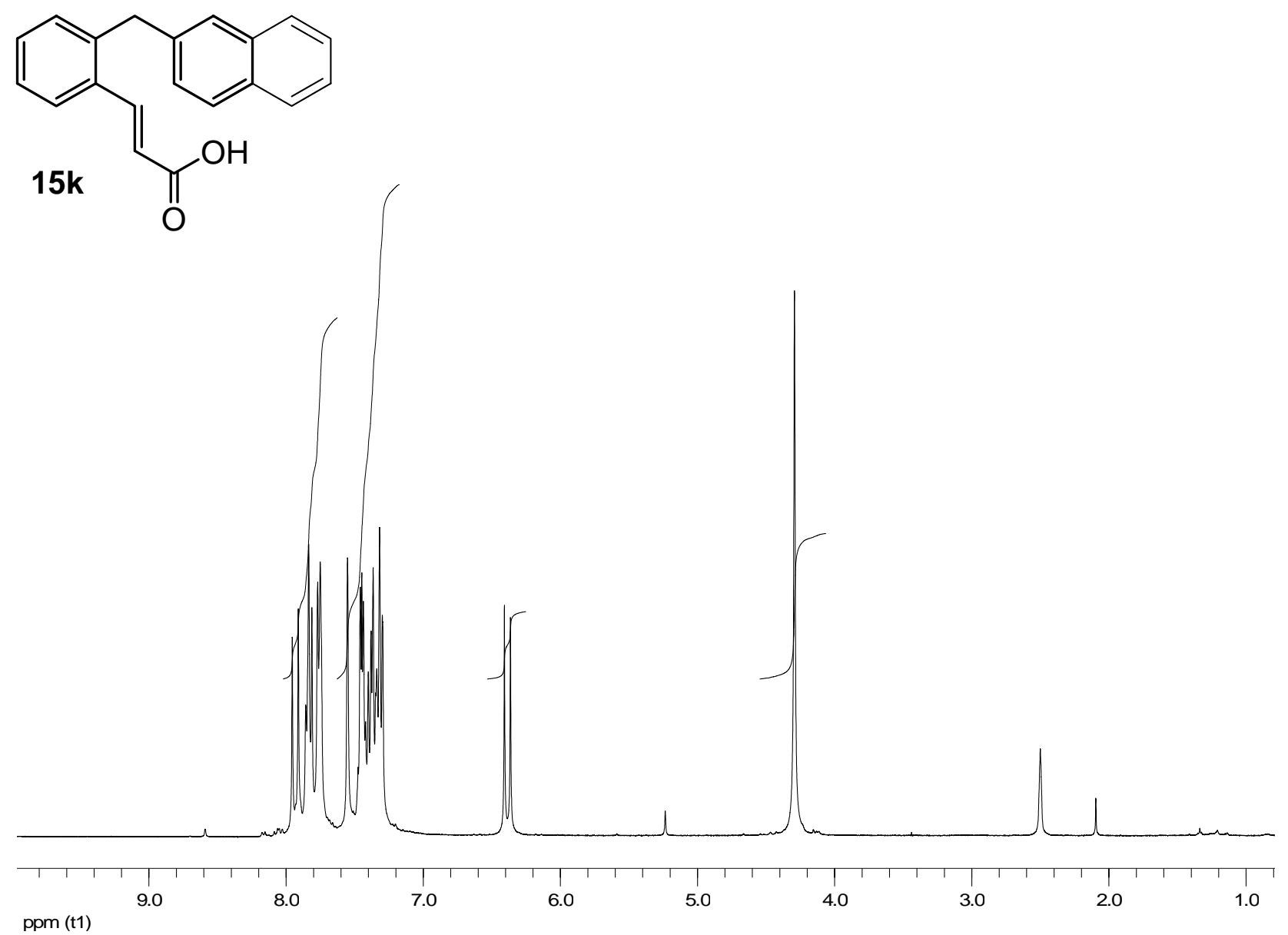

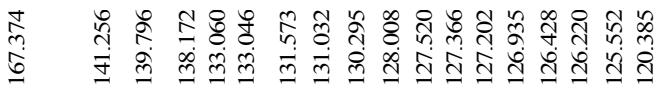
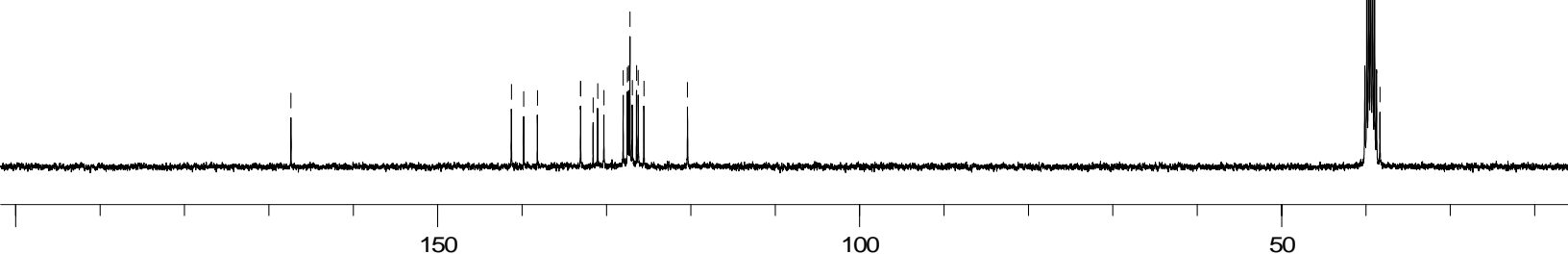

ppm (t1)

100

50 


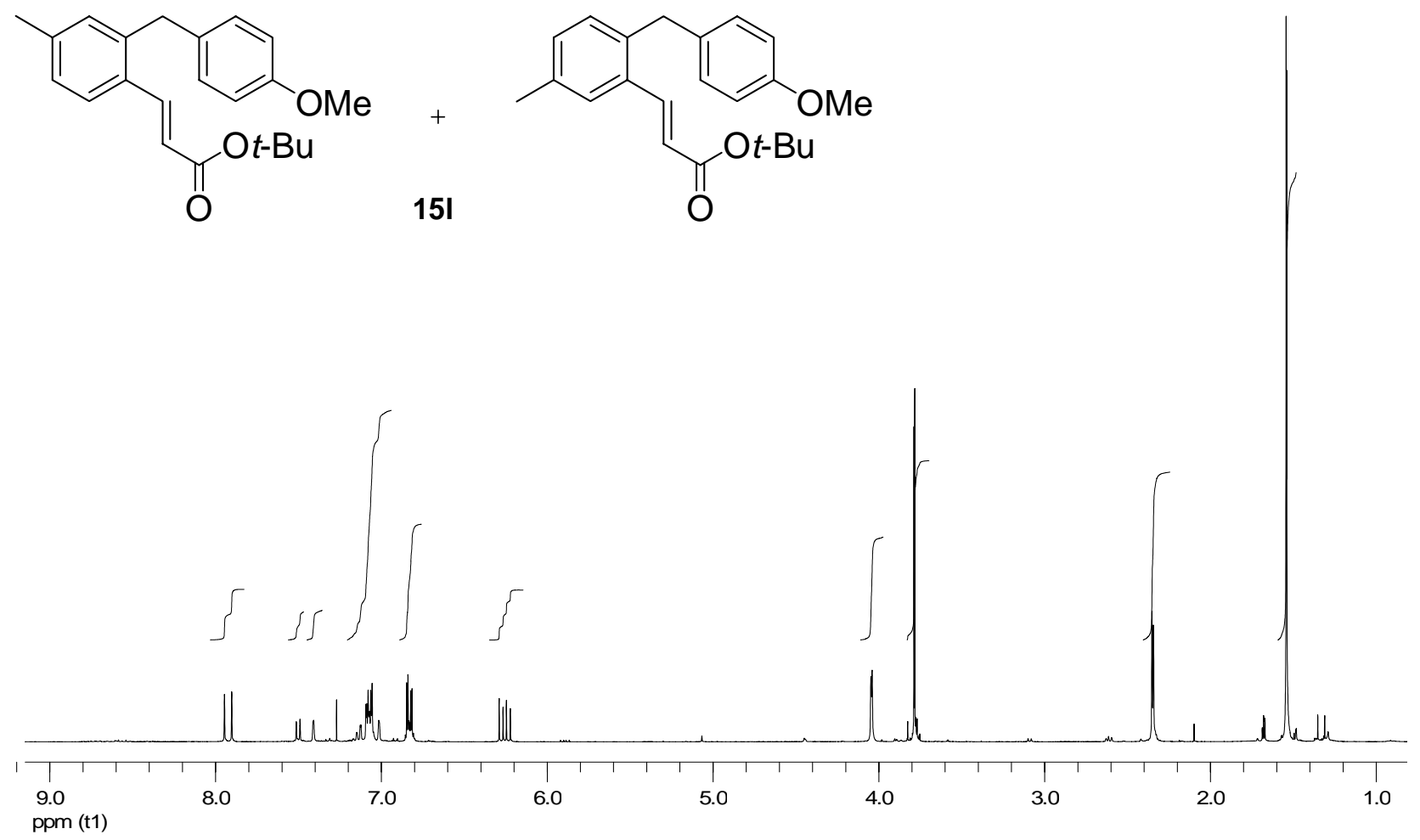

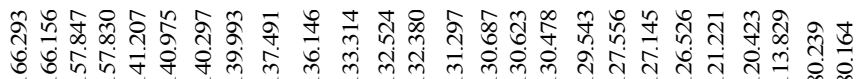
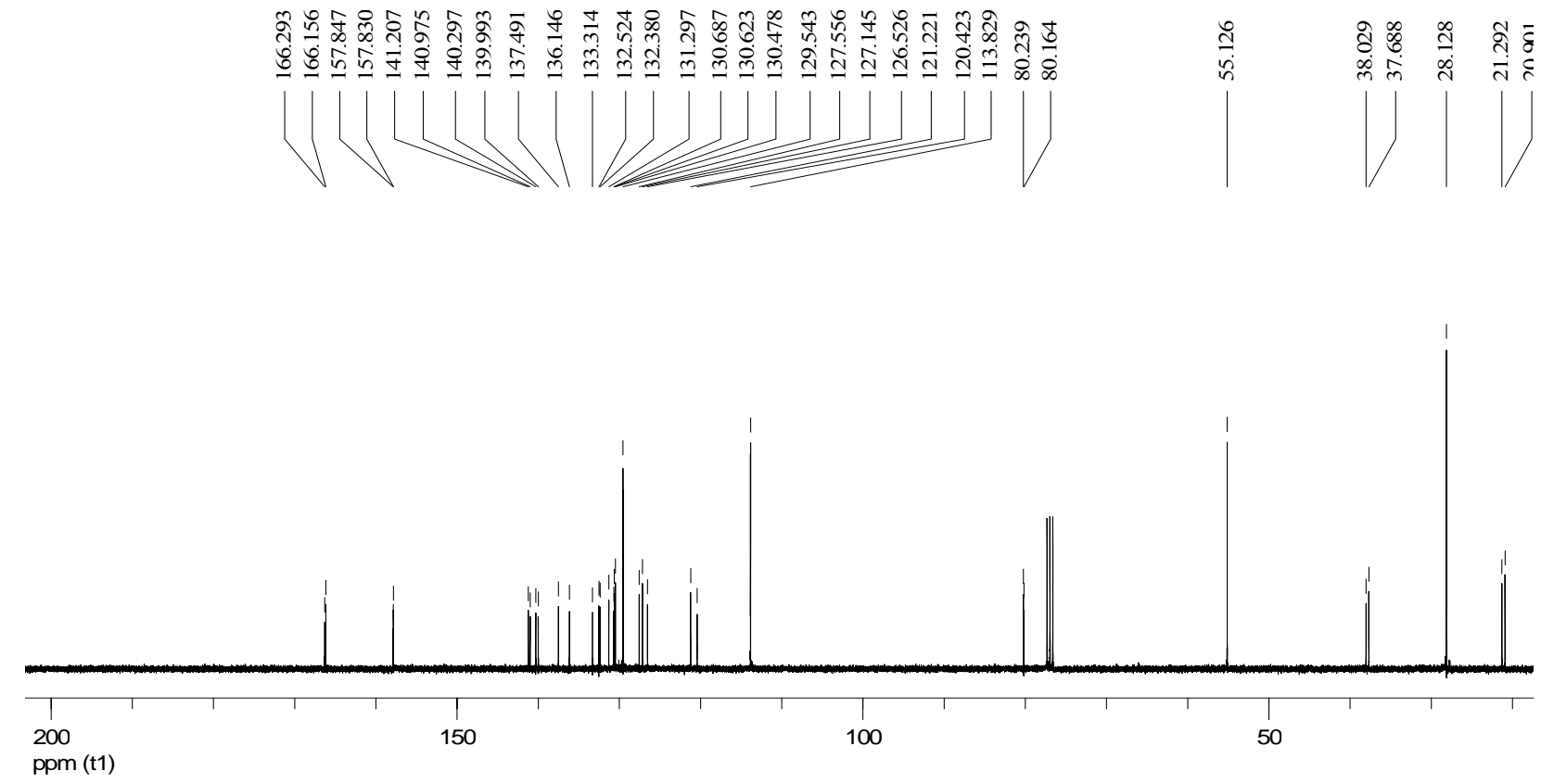